\title{
Limit Theorems for Conservative Flows on Multiple Stochastic Integrals
}

\author{
Shuyang Bai \\ bsy9142@uga.edu
}

March 15, 2021

\begin{abstract}
We consider a stationary sequence $\left(X_{n}\right)$ constructed by a multiple stochastic integral and an infinitemeasure conservative dynamical system. The random measure defining the multiple integral is nonGaussian, infinitely divisible and has a finite variance. Some additional assumptions on the dynamical system give rise to a parameter $\beta \in(0,1)$ quantifying the conservativity of the system. This parameter $\beta$ together with the order of the integral determines the decay rate of the covariance of $\left(X_{n}\right)$. The goal of the paper is to establish limit theorems for the partial sum process of $\left(X_{n}\right)$. We obtain a central limit theorem with Brownian motion as limit when the covariance decays fast enough, as well as a non-central limit theorem with fractional Brownian motion or Rosenblatt process as limit when the covariance decays slow enough.
\end{abstract}

Keywords: limit theorem; long-range dependence; infinite ergodic theory; multiple stochastic integral MCS Classification (2010): 60F17.

\section{Introduction}

For a stationary random sequence $\left(X_{k}\right)$ with finite variance, the notion long-range dependence (or long memory $)$ is typically associated with a slow power-law decay in the covariance $\operatorname{Cov}\left(X_{k}, X_{0}\right)$ as $k \rightarrow \infty$. An important perspective towards long-range dependence is to focus on its implication on limit theorems (cf. 28]). In particular, if one is interested in a limit theorem for sums, it is well-known that when $\left(X_{k}\right)$ has a finite variance marginally and is weakly dependent, one expects the following central limit theorem: as $n \rightarrow \infty$,

$$
\left(\frac{1}{n^{1 / 2}} \sum_{k=1}^{\lfloor n t\rfloor} X_{k}\right)_{t \geq 0} \Rightarrow(\sigma B(t))_{t \geq 0},
$$

where $\Rightarrow$ stands for a suitable mode of weak convergence, e.g., weak convergence in the Skorokhod space $D[0,1],(B(t))_{t \geq 0}$ is the standard Brownian motion, and $\sigma^{2}=\sum_{k} \operatorname{Cov}\left(X_{k}, X_{0}\right)$ with the summability often implied by the weak dependence condition imposed. On the other hand, when $\left(X_{k}\right)$ has long-range dependence where $\operatorname{Cov}\left(X_{k}, X_{0}\right)$ fails to be summable, the normalization $n^{1 / 2}$ in (1) is not strong enough to stablize the sum, and hence the central limit theorem (11) no longer holds. In fact in this case, one anticipates instead a limit theorem of the form:

$$
\left(\frac{1}{n^{H} L(n)} \sum_{k=1}^{\lfloor n t\rfloor} X_{k}\right)_{t \geq 0} \Rightarrow(Z(t))_{t \geq 0},
$$

where $L$ is a slowly varying function (e.g. a logarithm, cf. [5]), $H \in(1 / 2,1)$ is the so-called Hurst index, and $Z(t)$ is a $H$-self-similar (i.e., $\left.(Z(c t))_{t \geq 0} \stackrel{d}{=}\left(c^{H} Z(t)\right)_{t \geq 0}, c>0\right)$ with stationary increments which can 
be Gaussian or non-Gaussian. According to [28], the phase transition from (11) to (2) can be viewed as an indication of the long-range dependence in $\left(X_{k}\right)$.

An aspect of the limit theorem in (2) fundamentally different from (11) is the absence of universality of the limit. In (1) the limit process is always a Brownian motion regardless of the distribution of $\left(X_{k}\right)$. In contrast, the limit $Z(t)$ in (2) has many possibilities and often reflects some details in $\left(X_{k}\right)$. A limit theorem (2) under long-range dependence is often termed as a non-central limit theorem. A celebrated family of processes which are often found as limits in (2) are the Hermite processes [34, 8]. A standardized Hermite process can be represented as:

$$
Z_{p, \beta}(t)=a_{p, \beta} \int_{\mathbb{R}^{p}}^{\prime}\left(\int_{0}^{t} \prod_{j=1}^{p}\left(s-x_{j}\right)_{+}^{\beta / 2-1} d s\right) W\left(d x_{1}\right) \ldots W\left(d x_{p}\right), \quad t \geq 0,
$$

where $\int_{\mathbb{R}^{p}}^{\prime} \cdot W\left(d x_{1}\right) \ldots W\left(d x_{p}\right)$ denotes the multiple Wiener-Itô integral with $W$ being a Gaussian random measure with Lebesgue control measure,

$$
1-\frac{1}{p}<\beta<1
$$

and

$$
a_{p, \beta}=\left(\frac{(1-p(1-\beta) / 2)(1-p(1-\beta))}{p ! \mathrm{B}(\beta / 2,1-\beta)^{p}}\right)^{1 / 2}
$$

is a constant which makes $\operatorname{Var}\left[Z_{p, \beta}(1)\right]=1$ (see, e.g., [25, Proposition 4.4.2]). Here $\mathrm{B}(x, y)=\int_{0}^{1} z^{x-1}(1-$ $z)^{y-1} d z, x, y>0$, denotes the beta function. The process $Z_{p, \beta}(t)$ has Hurst index $H=1-p(1-\beta) / 2$, which is also known as the fractional Brownian motion when $p=1$ and the Rosenblatt process when $p=2$.

Recently, there has been some interest in studying limit theorems for a stationary sequence $\left(X_{k}\right)$ constructed through embedding an infinite-measure dynamical system in a (multiple) stochastic integral. Here we only minimally describe the necessary ingredients and leave the precise definitions to Section 2 , Suppose $(E, \mathcal{E}, \mu)$ is a measure space where $\mu$ is a $\sigma$-finite infinite measure. Fix a subspace $A \in \mathcal{E}$ satisfying $\mu(A)<\infty$. Let $T: E \rightarrow E$ be a $\mu$-preserving transform. Some additional ergodic-theoretic assumptions need to be imposed on the dynamical system $(E, \mathcal{E}, \mu, T)$ and the set $A$. They give rise to a parameter $\beta \in(0,1)$ (it will be identified with $\beta$ in (4) in the context of limit theorems), which loosely speaking, characterizes the frequency of the visits of the flow $\left\{T^{n}\right\}$ to $A$. Suppose $M$ is a symmetric infinitely-divisible random measure with control measure $\mu$. For a suitable measurable function $f: E^{p} \rightarrow \mathbb{R}$ which has support within $A^{p}$, we can define a stationary sequence by

$$
X_{k}=\int_{E^{p}}^{\prime} f\left(T^{k} x_{1}, \ldots, T^{k} x_{p}\right) M\left(d x_{1}\right) \ldots M\left(d x_{p}\right), \quad k \in \mathbb{Z}_{+},
$$

where the prime' indicates the exclusion of the diagonals $x_{i}=x_{j}, i \neq j$, in the multiple integral. The strength of the dependence in $\left(X_{k}\right)$ is controlled by the parameter $\beta$ and the order $p$, whereas the heaviness of the tails of $X_{k}$ is controlled by the random measure $M$. See [33] for a general theory of multiple integrals with respect to a symmetric infinitely-divisible random measure. We shall also provide a brief introduction in Section 2.2 below.

Initiated from the work of Owada and Samorodnitsky 23], the focus had been mainly on the case where $p=1$ and $M$ (and thus $X_{k}$ ) has an infinite variance [22, 14, 28, 21, 19, 30, 7, 6]. Limit theorems for sums of $\left(X_{k}\right)$ involving a general integral order $p$ were recently considered when $M$ is Gaussian [3] and when $M$ is infinitely divisible without a Gaussian component and has an infinite variance [4]. See Table1 1 for a crude classification of the existing results on limit theorems for sums.

In this paper, we shall consider limit theorems for sums of $\left(X_{k}\right)$ in (5) where $M$ is a finite-variance symmetric infinitely divisible random measure without a Gaussian component. We shall establish a central limit theorem when $\beta<1-1 / p$ and $p \in \mathbb{Z}_{+}$with a Brownian motion as limit, as well as a non-central limit theorem with a Hermite process in (3) as limit when $\beta>1-1 / p$ and $p=1$ or 2 . We note that a non-central limit theorem when $p=1$ has been considered in [28, Section 9.4] for a type of dynamical 


\begin{tabular}{|c|c|c|}
\hline Assumption on $M$ & $p=1$ & $p \geq 2$ \\
\hline Infinite variance & {$[23,14]$} & {$[4]($ only when $\beta>1-1 / p)$} \\
\hline Gaussian & {$[3]$} & {$[3]$} \\
\hline Finite variance non-Gaussian & {$[28]$, current work } & current work (when $\beta>1-1 / p$ or $p=2, \beta>1 / 2)$ \\
\hline
\end{tabular}

Table 1: Summary of existing literature on limit theorems for sums regarding the model (5). The classification presented here is crude and does not reflect many special assumptions and restrictions in these studies.

systems constructed by null-recurrent Markov chains, for which the proof relies on the infinite divisibility of the single integral and thus do not seem to apply to the case where $p=2$. On the other hand, while the results are analogs of some of those in [3] ; however, due to the absence of Gaussianity, the proof techniques are different here. Our proof strategy involves first an approximation of $M$ by a compound Poisson random measure with finite moments, and then a subtle execution of the method of moments. The case $\beta>1-1 / p$ and $p \geq 3$, however, cannot be concluded using the method of moments, and is left as a conjecture.

The paper is organized as follows. Section 2 prepares the background on infinite ergodic theory and multiple stochastic integrals. Section 3 contains the main results. Section 4 includes the proofs of the main results.

\section{Preliminaries}

First, we address some notation which will be used throughout the paper. For two positive sequences $a_{n}$ and $b_{n}$, we write $a_{n} \sim b_{n}$ to mean $\lim _{n} a_{n} / b_{n}=1$. For $a \in \mathbb{R}$, the notion $\operatorname{RV}_{\infty}(a)$ denotes the class of positive functions defined on $\mathbb{Z}_{+}$or $\mathbb{R}_{+}$, which are regularly varying with index $a$ at infinity (cf. Bingham et al. [5]). Write $\lfloor x\rfloor=\sup \{n \in \mathbb{Z}: n \leq x\}, x \in \mathbb{R}$. For a function $f: E \rightarrow \mathbb{R}$, we set $\operatorname{supp}(f)=\{x \in E: f(x) \neq 0\}$. The gamma function is $\Gamma(x)=\int_{0}^{\infty} u^{x-1} e^{-u} d u, x>0$. For a measure space $(E, \mathcal{E}, \mu)$, the notation $\left(E^{p}, \mathcal{E}^{p}, \mu^{p}\right)$ denotes its $p$-product measure space, $p \in \mathbb{Z}_{+}$. For an integrable or non-negative measurable function $f$ defined on $(E, \mathcal{E}, \mu)$, we use $\mu(f)$ to denote its integral $\int_{E} f d \mu$. For a finite ordered sequence $I=\left(i_{1}, \ldots, i_{p}\right) \in \mathbb{Z}_{+}^{p}$, $i_{1}<\ldots<i_{p}$, and $\left(a_{i}\right)_{i \in \mathbb{Z}_{+}}$, we write $a_{I}=\left(a_{i_{1}}, \ldots, a_{i_{p}}\right)$. This ordered sequence $I$ is at times treated as a subset of $\mathbb{Z}_{+}$as well.

\subsection{Background from infinite ergodic theory}

We introduce some necessary backgrounds from the infinite ergodic theory, for which the main reference is 1].

Let $(E, \mathcal{E}, \mu)$ be a measure space where $\mu$ is a $\sigma$-finite measure with $\mu(E)=\infty$. Suppose that $T: E \rightarrow E$ is a measure-preserving transform, namely, $T$ is measurable and $\mu\left(T^{-1} B\right)=\mu(B)$ for any $B \in \mathcal{E}$. We shall always make the following two basic assumptions:

- $T$ is ergodic, namely, $T^{-1} B=B \bmod \mu$ implies either $\mu(B)=0$ or $\mu\left(B^{c}\right)=0$;

- $T$ is conservative, namely, for any $B \in \mathcal{E}$ with $\mu(B)>0$, we have $\sum_{k=1}^{\infty} 1_{B}\left(T^{k} x\right)=\infty$ for a.e. $x \in B$.

These two assumptions combined are equivalent to the following statement ([1, Proposition 1.2.2]): for any $B \in \mathcal{E}$ with $\mu(B)>0$, we have

$$
\sum_{k=1}^{\infty} 1_{B}\left(T^{k} x\right)=\infty \quad \text { for a.e. } x \in E
$$

The dual (or Perron-Frobenius or transfer) operator $\widehat{T}$ of $T$ is defined as

$$
\widehat{T}: L^{1}(\mu) \rightarrow L^{1}(\mu), \quad \widehat{T} f=\frac{d\left(\mu_{f} \circ T^{-1}\right)}{d \mu},
$$


where the signed measure $\mu_{f}(B):=\int_{B} f d \mu, B \in \mathcal{E}$. The dual operator $\widehat{T}$ is characterized by the dual property:

$$
\int_{E} f \cdot(g \circ T) d \mu=\int_{E}(\widehat{T} f) \cdot g d \mu
$$

for any $f \in L^{1}(\mu)$ and $g \in L^{\infty}(\mu)$. It is useful to note the probabilistic interpretation of $\widehat{T}$ : if $f$ is the density with respect to $\mu$ of a random element $X$ taking value in $E$, then $\widehat{T} f$ is the density with respect to $\mu$ of the transformed random element $T X$. To see this, one can take $g$ to be an indicator function $1_{B}$, $B \in \mathcal{E}$, and then the dual property above becomes $P(T X \in B)=\int_{T^{-1} B} f d \mu=\int_{B} \widehat{T} f d \mu$. In addition, the characterization in (6) has an equivalent formulation using the dual operator (Proposition 1.3.2 Aaronson [1]): for any non-negative $f \in L^{1}(E, \mathcal{E}, \mu)$ satisfying $\mu(f)=\int_{E} f d \mu>0$, we have

$$
\sum_{k=1}^{\infty} \widehat{T}^{k} f=\infty \quad \text { a.e.. }
$$

The following assumption was proposed in Bai et al. [4] combining the ideas from Krickeberg [18] and literature on infinite ergodic theory such as Kesseböhmer and Slassi 17]. A similar framework was also used in Bai and Taqqu [3]. We shall adopt the following convention throughout: any function defined on a subspace (e.g. A) will be extended to the full space (e.g. E) by assuming zero value outside the subspace, whenever necessary.

Assumption 1. Let the dynamical system $(E, \mathcal{E}, \mu, T)$ be as above. There exists $A \in \mathcal{E}$ with $\mu(A) \in(0, \infty)$, so that $A$ is a Polish space with $\mathcal{E}_{A}:=\mathcal{E} \cap A$ being its Borel $\sigma$-field. In addition, there exists a positive rate sequence $\left(b_{n}\right)$ is regularly varying:

$$
\left(b_{n}\right) \in \mathrm{RV}_{\infty}(1-\beta), \quad \beta \in(0,1),
$$

and

$$
\lim _{n} b_{n} \widehat{T}^{n} g(x)=\mu(g)=\int_{E} g d \mu=\int_{A} g d \mu \quad \text { uniformly for a.e. } x \in A
$$

for all bounded and $\mu$-a.e. continuous $g$ on $A$ (i.e., the discontinuity set of $g$ has $\mu$ measure zero), where for simplicity we still use $\mu$ to denote its restriction to $\mathcal{E}_{A}$. (The last equality in (10) is due to that $g$ has its support within $A$ by the convention made before Assumption 1.)

Assumption 1 implies a rate of order $\mathrm{RV}_{\infty}(\beta)$ for the divergence in (8) over the subspace $A$, and hence $\beta$ introduced in Assumption 1 may be viewed as a parameter quantifying the conservativity of the system. The relation (10) in general cannot be extended to an arbitrary integrable function $g$ on $A$ due to the existence of weakly wandering sets [11]. Under the assumptions imposed so far, the sequence $\left(b_{n}\right)$ is also related to the wandering sequence:

$$
w_{n}=\mu\left(\bigcup_{k=1}^{n} T^{-k} A\right),
$$

through the following relation:

$$
b_{n} \sim \Gamma(\beta) \Gamma(2-\beta) w_{n}
$$

as $n \rightarrow \infty$. The relation (12) can be found in Proposition 3.1 of Kesseböhmer and Slassi 17]. In particular, as with the current paper, ergodicity and conservativity have been assumed throughout Kesseböhmer and Slassi [17], and Assumption 1 above is implied by their "uniform return" assumption imposed in their Definition 3.1 .

Remark. Specific examples of dynamical system $(E, A, \mathcal{E}, \mu, T)$ satisfying Assumption 1, which involve interval maps with indifferent fixed points and null-recurrent Markov chains, can be found in 4, Section 4.3]. We shall omit repeating these concrete examples in this paper. See also [17, 10, 20] for more examples and theories related to Assumption 1 . 
Assumption 1 implies the following "mixing-type" relation which essentially determines the covariance decay of $\left(X_{k}\right)$ in (5).

Lemma 2.1. Suppose $f_{1}$ and $f_{2}$ are bounded and $\mu^{p}$-a.e. continuous functions on $A^{p}$ (for simplicity we still use $\mu^{p}$ to denote its restriction to $\mathcal{E}_{A}^{p}$ ). Then

$$
\int_{E^{p}} f_{1}\left(T^{n} x_{1}, \ldots, T^{n} x_{p}\right) f_{2}\left(x_{1}, \ldots, x_{p}\right) \mu\left(d x_{1}\right) \ldots \mu\left(d x_{p}\right)=\mu^{p}\left(\left(f_{1} \circ T_{p}^{n}\right) \cdot f_{2}\right) \sim b_{n}^{-p} \mu^{p}\left(f_{1}\right) \mu^{p}\left(f_{2}\right)
$$

as $n \rightarrow \infty$, where

$$
T_{p}:=T \times \ldots \times T
$$

is the Cartesian product transform.

Proof. For any $B_{1}, B_{2} \in \mathcal{E}_{A}$ such that $1_{B_{1}}$ and $1_{B_{2}}$ are $\mu$-a.e. continuous functions on $A$ (namely, $\mu\left(\partial B_{1}\right)=$ $\mu\left(\partial B_{2}\right)=0$, where $\partial B$ denotes the boundary of a set $B \subset A$ ), using the dual property of $\widehat{T}$ in (7), the uniform convergence in (10) implies that

$$
\mu\left(B_{1} \cap T^{-n} B_{2}\right)=\int_{E} 1_{B_{1}} \cdot\left(1_{B_{2}} \circ T^{n}\right) d \mu=\int_{A}\left(\widehat{T}^{n} 1_{B_{1}}\right) \cdot 1_{B_{2}} d \mu \sim b_{n}^{-1} \mu\left(B_{1}\right) \mu\left(B_{2}\right)
$$

as $n \rightarrow \infty$. This verifies the mixing relation in [3, Equation (6)] and hence also [3, Assumption 2.1] after noting that $\left(b_{n}\right)$ here plays the same role as $\left(\rho_{n}\right)$ in [3]. (Strictly speaking, $\left(\rho_{n}\right)$ in [3] was restricted to a subclass of $\operatorname{RV}_{\infty}(1-\beta)$, although this restriction can be easily relaxed.) In view of [3, Proposition 2.6], we know that [3, Assumption 2.1] holds if the system $(E, \mathcal{E}, \mu, T, A)$ is replaced by the product system $\left(E^{p}, \mathcal{E}^{p}, \mu^{p}, T_{p}, A^{p}\right)$. With [3, Proposition 2.5] applied to the product system, the conclusion of this lemma follows (or see directly [3, Equation (11)]).

\subsection{Random measure and multiple stochastic integrals}

We shall first provide a brief introduction to an infinitely divisible random measure. For a formal definition, see [28, Section 3.2]. Let $(E, \mathcal{E}, \mu)$ be a measure space where $\mu$ is $\sigma$-finite and atomless. An infinitely divisible (signed) random measure $M$ on $(E, \mathcal{E})$ with control measure $\mu$ can be viewed as an infinitely divisible stochastic process with index set $\mathcal{E}_{0}:=\{B \in \mathcal{E}: \mu(B)<\infty\}$. The random measure $M$ is independently scattered and $\sigma$-additive: for any disjoint $B_{n} \in \mathcal{E}_{0}, n \in \mathbb{Z}_{+}$, the random variables $M\left(B_{1}\right), M\left(B_{2}\right), \ldots$ are independent, and if in addition $\cup_{n=1}^{\infty} B_{n} \in \mathcal{E}_{0}$, then $M\left(\cup_{n=1}^{\infty} B_{n}\right)=\sum_{n=1}^{\infty} M\left(B_{n}\right)$ a.s.. In view of these properties, the law of $M$ is completely specified by the law of the marginal distributions of $M(B), B \in \mathcal{E}_{0}$. As an infinitely divisible process, it is well-known that $M$ can be decomposed into a Gaussian component and a Poisson component. We shall work with an infinitely divisible random measure $M$ with only the Poisson component present. Below is the precise assumption on $M$ which defines the stationary sequence in (5).

Assumption 2. $M$ is an infinitely divisible random measure $(E, \mathcal{E}, \mu)$ whose law is specified by

$$
\mathbb{E} e^{i u M(B)}=\exp \left(-\mu(B) \int_{\mathbb{R}}(1-\cos (u y)) \rho(d y)\right), \quad u \in \mathbb{R},
$$

where $B \in \mathcal{E}_{0}$, and $\rho$ is a symmetric Lévy measure on $\mathbb{R}$ satisfying $\rho(\{0\})=0$ with a unit second moment:

$$
\int_{\mathbb{R}} x^{2} \rho(d x)=1
$$

These assumptions on $M$ are similar to those in [4], except that in this paper $M$ has a finite variance. The symmetry of $\rho$ implies that

$$
\mathbb{E} M(B)=0, B \in \mathcal{E}_{0}
$$


and the standardization (15) implies

$$
E M(B)^{2}=-\left.\frac{d^{2}}{d u^{2}} \ln \mathbb{E} e^{i u M(B)}\right|_{u=0}=\mu(B) \int_{\mathbb{R}} x^{2} \rho(d x)=\mu(B), B \in \mathcal{E}_{0} .
$$

We shall also need the following generalized inverse of the tail Lévy measure:

$$
\rho^{\leftarrow}(y)=\inf \{x>0: \rho((x, \infty)) \leq y / 2\}, \quad y>0 .
$$

The following relation between the generalized inverse and the moments of the Lévy measure will be useful.

Lemma 2.2. We have for any $r>0$ that

$$
\int_{\mathbb{R}} x^{r} \rho(d x)=\int_{0}^{\infty} \rho^{\leftarrow}(y)^{r} d y
$$

Proof. By the symmetry of $\rho$, Fubini and the equivalence $y / 2<\rho((x, \infty)) \Longleftrightarrow x<\rho^{\leftarrow}(y), x, y>0$, we have

$$
\begin{aligned}
\int_{\mathbb{R}} x^{r} \rho(d x) & =2 \int_{(0, \infty)} x^{r} \rho(d x)=2 \int_{0}^{\infty} r x^{r-1} \rho((x, \infty)) d x=\int_{0}^{\infty} r x^{r-1} d x \int_{0}^{\infty} 1_{\{y / 2<\rho((x, \infty))\}} d y \\
& =\int_{0}^{\infty} d y \int_{0}^{\rho^{\leftarrow}(y)} r x^{r-1} d x=\int_{0}^{\infty} \rho^{\leftarrow}(y)^{r} d y .
\end{aligned}
$$

For a function $f \in L^{2}\left(E^{p}, \mathcal{E}^{p}, \mu^{p}\right), p \in \mathbb{Z}_{+}$, the (off-diagonal) multiple integral

$$
I_{p}(f)=\int_{E^{p}}^{\prime} f\left(x_{1}, \ldots, x_{p}\right) M\left(d x_{1}\right) \ldots M\left(d x_{p}\right)
$$

can be defined using a classical approach orignated from [12]: first for $f=1_{B_{1} \times \ldots \times B_{p}}$ with disjoint $B_{i} \in \mathcal{E}_{0}$, $i=1, \ldots, p$, define $I_{p}(f)=M\left(B_{1}\right) \ldots M\left(B_{p}\right)$. Then extend the definition to a linear combination of such functions, and finally to a general $f \in L^{2}$ by a $L^{2}$ approximation. It is important, as indicated by the prime ', that the diagonal set $D=\left\{\left(x_{1}, \ldots, x_{p}\right) \in E^{p}: x_{i}=x_{j}, i \neq j\right\}$ is excluded in the integration. In view of this, one may always treat the integrand $f$ as $f 1_{D^{c}}$. See [24, Section 5.4] for more details. Because of the invariance of the integral with respect to the permutation of the variables of $f$, one can often assume without loss of generality that $f$ is symmetric, that is, its value is invariant with respect to any permutation of its variables. For symmetric $f_{1}, f_{2} \in L^{2}\left(E^{p}, \mathcal{E}^{p}, \mu^{p}\right)$, we have the $L^{2}$ isometry property:

$$
\mathbb{E} I_{p_{1}}\left(f_{1}\right) I_{p_{2}}\left(f_{2}\right)= \begin{cases}p ! \mu^{p}\left(f_{1} f_{2}\right) & \text { if } p_{1}=p_{2}=: p \\ 0 & \text { if } p_{1} \neq p_{2} .\end{cases}
$$

Alternatively, the multiple integral may be constructed through a series representation of the symmetric infinitely divisible random measure $M$ without a Gaussian component (see, e.g., 33]). Such a construction is used in a coupling argument in Section 4.1 and in the proof of tightness in $D[0,1]$ in Section 4.2 If in addition $\rho(\mathbb{R})<\infty$, the multiple integral may also be expressed through a compound Poisson representation of $M$ (see Section 4.1), which we shall use to facilitate the computation of moments.

\section{Main results}

In this section we state the main results. Throughout this section we shall make the following assumptions: 
- $(E, \mathcal{E}, \mu)$ is an atomless $\sigma$-finite infinite-measure space;

- $(E, \mathcal{E}, \mu, T)$ is ergodic and conservative with a distinguished subspace $A$ satisfying Assumption 1 .

- The random measure $M$ satisfies Assumption 2

- The stationary sequence $\left(X_{k}\right)$ is as in (5), where $f$ is a symmetric bounded and $\mu^{p}$-a.e. continuous function on $A^{p}$ (extended to $\left(A^{p}\right)^{c}$ by taking zero value there).

Note that the $\mu^{p}$-a.e. continuity of $f$ is with respect to the product topology of the subspace $A$ in Assumption 1. Such $f$ is always in $L^{2}\left(\mu^{p}\right)$ since it is bounded and $\operatorname{supp}(f) \subset A^{p}$ where $\mu^{p}\left(A^{p}\right)=\mu(A)^{p}<\infty$, and hence $\left(X_{k}\right)$ is well-defined in view of Section 2.2 .

We first clarify the memory property of $\left(X_{k}\right)$ implied by Assumption 1. Recall the notation $\mu^{p}(f)=$ $\int_{E^{p}} f d \mu^{p}$.

Corollary 3.1. We have as $k \rightarrow \infty$,

$$
\mathbb{E}\left[X_{k} X_{0}\right]=p ! \mu^{p}\left(\left(f \circ T_{p}^{k}\right) \cdot f\right) \sim p ! \mu^{p}(f)^{2} b_{k}^{-p} \in \operatorname{RV}_{\infty}(p(\beta-1)) .
$$

Corollary 3.1 follows from (19) and Lemma 2.1. Depending on whether $p(\beta-1)<-1$ or $p(\beta-1)>-1$, the covariance $\mathbb{E}\left[X_{k} X_{0}\right]$ is summable or not. This corresponds to a classical distinction between short-range dependence and long-range dependence. In particular, in the short-range dependence regime $p(\beta-1)<-1$, it can be shown that $\operatorname{Var}\left(\sum_{k=1}^{n} X_{k}\right)$ scales linearly as $n \rightarrow \infty$, whereas in the long-range dependence regime $p(\beta-1)>-1$, the variance $\operatorname{Var}\left(\sum_{k=1}^{n} X_{k}\right)$ scales super-linearly. Hence the order of normalization in limit theorems need to be chosen differently in these two situations. See, e.g., [25, Chapter 2], for more details.

Theorem 3.2. If $p(\beta-1)<-1$ (so necessarily $p \geq 2$ ), then as $n \rightarrow \infty$,

$$
\left(\frac{1}{n^{1 / 2}} \sum_{k=1}^{\lfloor n t\rfloor} X_{k}\right)_{t \in[0,1]} \stackrel{\text { f.d.d. }}{\longrightarrow}(\sigma B(t))_{t \in[0,1]},
$$

where $\stackrel{\text { f.d.d. }}{\longrightarrow}$ stands for convergence of finite-dimensional distributions, $B$ is a standard Brownian motion, and

$$
\sigma^{2}=\sum_{k=-\infty}^{\infty} \mathbb{E}\left[X_{k} X_{0}\right]=\sum_{k=-\infty}^{\infty} p ! \mu^{p}\left(\left(f \circ T_{p}^{k}\right) \cdot f\right) .
$$

If in addition, $\int_{\mathbb{R}} x^{4} \rho(d x)<\infty$, then $\stackrel{\text { f.d.d. }}{\longrightarrow}$ can be replaced by weak convergence in $D[0,1]$ with the uniform metric.

The proof of Theorem 3.2 can be found in Section 4 We believe that for the convergence in $D[0,1]$, the assumption of a finite fourth moment is an artifact of our proof technique and may be relaxed. We also note that in the case $p(\beta-1)=-1$, we anticipate a central limit theorem similar to (10) to hold with Brownian motion as limit, although depending on the slowly varying factor in (9), an additional slowly varying factor may appear in the normalization in (11). Treating the case $p(\beta-1)=1$ requires some technical but non-essential modification of the proof below for Theorem 3.2, which we shall omit in this paper.

Theorem 3.3. If $p(\beta-1) \in(-1,0)$, and $p=1$ or 2 , then as $n \rightarrow \infty$,

$$
\left(\frac{1}{a_{n}} \sum_{k=1}^{\lfloor n t\rfloor} X_{k}\right)_{t \in[0,1]} \Rightarrow\left(\mu^{p}(f) Z_{p, \beta}(t)\right)_{t \in[0,1]}
$$


where $\Rightarrow$ stands for weak convergence in $D[0,1]$ with the uniform metric, $\mu^{p}(f)=\int_{A^{p}} f d \mu^{p}$, the process $Z_{p, \beta}$ is the standard Hermite process as in (3) (i.e., the fractional Brownian motion if $p=1$, and the Rosenblatt process if $p=2$ ), the normalization sequence

$$
\left(a_{n}\right)=\left(\left(\frac{1}{(1-p(1-\beta) / 2)(1-p(1-\beta)) p !}\right)^{1 / 2} \frac{n}{b_{n}^{p / 2}}\right) \in \operatorname{RV}_{\infty}(1-p(1-\beta) / 2)
$$

where $\left(b_{n}\right)$ is as in (10) and $1-p(1-\beta) / 2 \in(1 / 2,1)$.

The proof of Theorem 3.3 can be found in Section 4. We mention that for $p=1$, a result similar to Theorem 3.3 has been considered in [28, Theorem 9.4.7]. There the dynamical system $(E, \mathcal{E}, \mu, T)$ was constructed using the path space of a null-recurrent Markov chain and its infinite invariant measure. The proof exploited the infinite divisibility of a single stochastic integral.

The reason we can only include cases $p=1$ and 2 in Theorem 3.3 is because moment determinacy either ceases to hold or is unknown for the limit law when $p \geq 3$ (see 32], and a simple explanation is that higher $p$ leads to heavier tails of a multiple Gaussian integral, see e.g., [13, Theorem 6.12]). Hence our proof based on the method of moments cannot conclude the cases where $p \geq 3$. Nevertheless, we expect the following conjecture to hold.

Conjecture 3.4. The conclusion of Theorem 3.3 continues to hold if $p \geq 3$.

The appearance of Hermite processes as non-central limits may be better physically understood in view of the new representations of Hermite processes recently obtained in 2], which involve the local time of intersecting stable regenerative sets. Although the moment calculation performed in this paper (Proposition 4.6 below) cannot conclude Conjecture 3.4 yet it provides a compelling evidence. A conclusive proof for $p \geq 3$ may need to exploit the local time representations in [2].

We also mention that it is natural to consider an extension of the results in the paper to the case where the infinitely divisible random measure $M$ has both Gaussian and non-Gaussian components. Indeed when $p=1$, such an extension is straightforward since one can decompose the single integral into two independent components. When $p \geq 2$, however, such an independent decomposition no longer holds. Hence the extension does not follow from a straightforward combination of the results in [3] and those in the current work. This problem is left for a future work.

\section{Proofs of the main results}

First we provide a summary of the proof strategy. We first establish a reduction result which enables us to replace the random measure $M$ in Assumption 2 by one with a finite Lévy measure $\rho_{0}$ whose moments of all orders exist. This reduction result is justified through a coupling argument based on series representations of infinitely divisible random measures without Gaussian components (see Lemma 4.3 below). With such a finite Lévy measure $\rho_{0}$, the corresponding random measure admits a compound Poisson representation with all the moments available. We can hence approach the convergence of finite-dimensional distributions in Theorems 3.2 and 3.3 by the method of moments. The tightness in $D[0,1]$ in Theorem 3.2 is established via a fourth moment computation using the series representation. The tightness in Theorem [3.3. on the other hand, follows from a well-known argument.

Below throughout, we use $c$ to denote a generic positive constant, whose value may change from line to line.

\subsection{Reduction}

We shall follow the assumptions and notation in Section 3.

Let $\rho_{0}$ be a symmetric Lévy measure on $\mathbb{R}$ satisfying $\rho_{0}(\{0\})=0$ and

$$
\int_{\mathbb{R}}\left(1 \vee x^{2}\right) \rho_{0}(d x)<\infty .
$$


Then $\rho_{0}$ is integrable and hence the Lévy measure of a compound Poisson distribution. Set

$$
Q=\rho_{0}(\mathbb{R}) .
$$

Recall that $A$ is the distinguished subspace in Assumption 1 . For fixed $n \in \mathbb{Z}_{+}$, we set

$$
A_{n}=\bigcup_{k=1}^{n} T^{-k} A
$$

Note that in view of (11) we have $\mu\left(A_{n}\right)=w_{n} \in(0, \infty)$ since $\mu(A) \in(0, \infty)$. On the probability measure space

$$
\left(A_{n}, \mathcal{E}_{n}:=\mathcal{E} \cap A_{n}, \mu_{n}(\cdot):=\left.\mu\right|_{\mathcal{E}_{n}}(\cdot) / w_{n}\right),
$$

where $\left.\mu\right|_{\mathcal{E}_{n}}$ denotes the restriction of $\mu$ to $\mathcal{E}_{n}$, we define a random measure

$$
M_{1, n}(\cdot)=\sum_{i=1}^{N_{n}} Z_{i} \delta_{U_{i, n}}(\cdot)
$$

where $\delta_{x}$ is the delta measure at $x$ inE, $\left(U_{i, n}\right)$ are i.i.d. random elements taking value in $A_{n}$ with distribution $\mu_{n},\left(Z_{i}\right)$ are i.i.d. symmetric real random variables with distribution $\rho_{0}(\cdot) / Q$ whose moments of all orders exist,

$$
N_{n}:=N\left(Q \mu\left(A_{n}\right)\right)=N\left(Q w_{n}\right)
$$

with $(N(t))_{t \geq 0}$ being a unit-rate Poisson process, and $\left(U_{i, n}\right),\left(Z_{i}\right)$ and $(N(t))$ are all independent of each other. Using some well-known properties of the Poisson process, one can verify that $M_{1, n}$ is infinitely divisible and independently scattered (and it is obviously $\sigma$-additive). An elementary computation (see, e.g., 28, Example 3.1.1]) shows that the random measure $M_{1, n}$ satisfies (14) but with $\rho$ replaced by $\rho_{0}$.

Next, we introduce a second random measure on $\left(A_{n}, \mathcal{E}_{n}\right)$ by setting

$$
M_{2, n}(\cdot)=\sum_{i=1}^{\infty} \epsilon_{i} \rho_{0}^{\leftarrow}\left(\Gamma_{i} / w_{n}\right) \delta_{U_{i, n}}(\cdot)
$$

where $\left(\epsilon_{i}\right)$ are i.i.d. Rademacher random variables, $\Gamma_{i}=E_{1}+\ldots+E_{i}$ with $\left(E_{j}\right)$ i.i.d. standard exponential random variables, $\left(U_{i, n}\right)_{i=1, \ldots, n}$ are i.i.d. random elements taking value in $A_{n}$ with distribution $\mu_{n}$ as before, $\rho_{0}^{\leftarrow}$ is the generalized inverse of $\rho_{0}$ as defined in (17), and $\left(\epsilon_{i}\right),\left(E_{i}\right)$ and $\left(U_{i, n}\right)$ are independent. The form (26) is in general known as a series representation of an infinitely divisible process (e.g., 26], 28, Section 3.4]). It follows from [28, Theorem 3.4.3] (see also [27]) that $M_{2, n}$ on $\left(A_{n}, \mathcal{E}_{n}\right)$ is also an infinitely divisible random measure satisfying (14) with $\rho$ replaced by $\rho_{0}$, and hence

$$
M_{1, n}(\cdot) \stackrel{d}{=} M_{2, n}(\cdot)
$$

for fixed $n \in \mathbb{Z}_{+}$.

We introduce a third random measure $M_{3, n}$ defined as in (26) using the same $\left(\epsilon_{i}\right)$ and $\left(\Gamma_{i}\right)$ and $\left(U_{i, n}\right)$, except that $\rho_{0}^{\leftarrow}$ is replaced by $\rho^{\leftarrow}$. Then as above $M_{3, n}$ is an infinitely divisible random measure satisfying (14) and hence

$$
M_{3, n}(\cdot) \stackrel{d}{=} M(\cdot)
$$

where $M$ is as in Assumption 2 but restricted to the subspace $\left(A_{n}, \mathcal{E}_{n}\right)$.

Here we explain the reason we introduce these random measures. In particular, for an integrand $f$ : $E^{p} \rightarrow \mathbb{R}$ as described in Section 3 which is symmetric, bounded and has support within $A^{p}$, we introduce for $1 \leq k \leq n$ that

$$
X_{k, 1}^{(n)}:=\int_{E^{p}}^{\prime}\left(f \circ T_{p}^{k}\right)\left(x_{1}, \ldots, x_{p}\right) M_{1, n}\left(d x_{1}\right) \ldots M_{1, n}\left(d x_{p}\right)=p ! \sum_{I \in \mathcal{D}_{p}\left(N_{n}\right)}\left(\prod_{i \in I} Z_{i}\right)\left(f \circ T_{p}^{k}\right)\left(U_{I, n}\right),
$$


where $U_{I, n}=\left(U_{i_{1}, n}, \ldots, U_{i_{p}, n}\right)$, and

$$
\mathcal{D}_{p}(n):=\left\{I=\left(i_{1}, \ldots, i_{p}\right): 1 \leq i_{1}<\ldots<i_{p} \leq n\right\} .
$$

To obtain the second equality in (29), we have used the exclusion of the diagonals of the multiple integral and the symmetry of $f$. Meanwhile, we set for $1 \leq k \leq n$ that

$$
X_{k, 2}^{(n)}:=\int_{E^{p}}^{\prime}\left(f \circ T_{p}^{k}\right)\left(x_{1}, \ldots, x_{p}\right) M_{2, n}\left(d x_{1}\right) \ldots M_{2, n}\left(d x_{p}\right)=p ! \sum_{I \in \mathcal{D}_{p}}\left(\prod_{i \in I} \epsilon_{i} \rho_{0}^{\leftarrow}\left(\Gamma_{i} / w_{n}\right)\right)\left(f \circ T_{p}^{k}\right)\left(U_{I, n}\right),
$$

where

$$
\mathcal{D}_{p}:=\left\{I=\left(i_{1}, \ldots, i_{p}\right): 1 \leq i_{1}<\ldots<i_{p}\right\} .
$$

The multilinear series in (31) converges unconditionally a.s., namely, regardless of the order the terms are added, the series converges a.s. to the same limit (cf. 27, Section 1]). In view of (27) we have

$$
\left(X_{k, 1}^{(n)}\right)_{1 \leq k \leq n} \stackrel{d}{=}\left(X_{k, 2}^{(n)}\right)_{1 \leq k \leq n}
$$

At last, we let $X_{k, 3}^{(n)}$ be defined as (31) but with $M_{2, n}$ replaced by $M_{3, n}$, and in view of (28), we have

$$
\left(X_{k, 3}^{(n)}\right)_{1 \leq k \leq n} \stackrel{d}{=}\left(X_{k}\right)_{1 \leq k \leq n}
$$

where $\left(X_{k}\right)$ is as in (5). The idea is that by approximating $\rho$ with $\rho_{0}$, one can then approximate $\left(X_{k, 3}^{(n)}\right)_{1 \leq k \leq n}$ with $\left(X_{k, 2}^{(n)}\right)_{1 \leq k \leq n}$ (see Lemma 4.3 below for more details). This enables one to work with eventually $\left(X_{k, 1}^{(n)}\right)_{1 \leq k \leq n}$, whose form is more amenable to the computation of moments compared to $\left(X_{k, 2}^{(n)}\right)_{1 \leq k \leq n}$.

Next, we prepare some results which are useful for the main reduction lemma below. They will also be useful in the proofs by the method of moments later. For $I \in \mathcal{D}_{p}$ and $t \in[0,1]$, we define

$$
L_{n, I, t}=\sum_{k=1}^{\lfloor n t\rfloor}\left(f \circ T_{p}^{k}\right)\left(U_{I, n}\right) .
$$

Recall that when $\beta<1-1 / p$, the sum $\sum_{k} \mu^{p}\left(\left(f \circ T_{p}^{k}\right) \cdot f\right)$ converges due to Lemma 4.1

Lemma 4.1. When $\beta<1-1 / p$, we have for any $I \in \mathcal{D}_{p}$,

$$
\mathbb{E}\left[L_{n, I, t_{1}} L_{n, I, t_{2}}\right] \sim n w_{n}^{-p}\left(t_{1} \wedge t_{2}\right) \sum_{k=-\infty}^{\infty} \mu^{p}\left(\left(f \circ T_{p}^{k}\right) \cdot f\right)
$$

as $n \rightarrow \infty$. In addition for any $1 \leq m \leq n$,

$$
\mathbb{E}\left[\left(\sum_{k=1}^{m}\left(f \circ T_{p}^{k}\right)\left(U_{I, n}\right)\right)^{2}\right] \leq w_{n}^{-p} m \sum_{k=-\infty}^{\infty}\left|\mu^{p}\left(\left(f \circ T_{p}^{k}\right) f\right)\right| .
$$

Proof. For $-n \leq k \leq n$, set

$$
\gamma_{n}(k):=\mathbb{E}\left[\left(f \circ T_{p}^{k}\right)\left(U_{I, n}\right) \cdot f\left(U_{I, n}\right)\right]=w_{n}^{-p} \mu^{p}\left(\left(f \circ T_{p}^{k}\right) \cdot f\right) .
$$


Assume without loss of generality that $0<t_{1} \leq t_{2} \leq 1$. We now focus on the first claim. Using the invariance $\mu^{p}\left(T_{p}^{-1} \cdot\right)=\mu_{p}(\cdot)$, we have

$$
\begin{aligned}
\mathbb{E}\left[L_{n, I, t_{1}} L_{n, I, t_{2}}\right] & =\sum_{k_{1}=1}^{\left\lfloor n t_{1}\right\rfloor} \sum_{k_{2}=1}^{\left\lfloor n t_{2}\right\rfloor} \gamma_{n}\left(k_{2}-k_{1}\right)=\sum_{k_{1}=1}^{\left\lfloor n t_{1}\right\rfloor} \sum_{k_{2}=1}^{\left\lfloor n t_{1}\right\rfloor} \gamma_{n}\left(k_{2}-k_{1}\right)+\sum_{k_{1}=1}^{\left\lfloor n t_{1}\right\rfloor} \sum_{k_{2}=\left\lfloor n t_{1}\right\rfloor+1}^{\left\lfloor n t_{2}\right\rfloor} \gamma_{n}\left(k_{2}-k_{1}\right) \\
& =: \mathrm{I}_{n}+\mathrm{II}_{n},
\end{aligned}
$$

where the term $\mathrm{II}_{n}$ is understood as zero if $\left\lfloor n t_{1}\right\rfloor=\left\lfloor n t_{2}\right\rfloor$. By Lemma 2.1 we have $\mu^{p}\left(\left(f \circ T_{p}^{k}\right) \cdot f\right) \sim b_{k}^{-p} \mu^{p}(f)^{2}$ as $k \rightarrow \infty$ which belongs to $\operatorname{RV}_{\infty}(p(\beta-1))$ with $p(\beta-1)<-1$, and hence $\sum_{k=-\infty}^{\infty}\left|\mu^{p}\left(\left(f \circ T_{p}^{k}\right) \cdot f\right)\right|<\infty$. It then follows from [25, Lemma 5.4.4] that $\mathrm{II}_{n}=w_{n}^{-p} O(n)$ as $n \rightarrow \infty$. In addition, by rearranging the double sum,

$$
\mathrm{I}_{n}=n w_{n}^{-p} t_{1} \sum_{k=-\left\lfloor n t_{1}\right\rfloor}^{\left\lfloor n t_{1}\right\rfloor}\left(\frac{\left\lfloor n t_{1}\right\rfloor}{n t_{1}}-\frac{|k|}{n t_{1}}\right) \mu^{p}\left(\left(f \circ T_{p}^{k}\right) \cdot f\right) .
$$

The first claim follows if one shows that the sum above converges to $\sum_{k=-\infty}^{\infty} \mu^{p}\left(\left(f \circ T_{p}^{k}\right) \cdot f\right)$ as $n \rightarrow \infty$. This can be verified by the dominated convergence theorem since $\sum_{k=-\infty}^{\infty}\left|\mu^{p}\left(\left(f \circ T_{p}^{k}\right) \cdot f\right)\right|<\infty$.

Now we turn to the second claim. For any $1 \leq m \leq n$, we have

$$
\mathbb{E}\left[\left(\sum_{k=1}^{m}\left(f \circ T_{p}^{k}\right)\left(U_{I, n}\right)\right)^{2}\right]=m \sum_{k=-m}^{m}\left(1-\frac{|k|}{m}\right) \gamma_{n}(|k|) .
$$

The conclusion follows since $\left|\left(1-\frac{|k|}{m}\right) \gamma_{n}(|k|)\right| \leq\left|\gamma_{n}(|k|)\right|$ for $-m \leq k \leq m$.

Introduce for $q \geq 2$, a symmetric function $h_{q}^{(\beta)}$ which is a.e. defined on $(0,1)^{q}$ as

$$
h_{q}^{(\beta)}\left(x_{1}, \ldots, x_{q}\right)=\Gamma(\beta) \Gamma(2-\beta) \prod_{j=2}^{q}\left(x_{j}-x_{j-1}\right)^{\beta-1}, 0<x_{1}<\cdots<x_{q}<1 .
$$

Define also $h_{0}^{(\beta)}:=1$ and $h_{0}^{(\beta)}(x):=\Gamma(\beta) \Gamma(2-\beta)$. For $r \in \mathbb{Z}_{+}$, define

$$
\mathcal{I}(i)=\left\{\ell \in\{1, \ldots, r\}: i \in I_{\ell}\right\} .
$$

Lemma 4.2. [[4, Proposition 5.3].] When $\beta \in(1-1 / p, 1)$, we have for any $I_{1}, \ldots, I_{r} \in \mathcal{D}_{p}, t_{1}, \ldots, t_{r} \in[0,1]$ that

$$
\lim _{n} \mathbb{E} \prod_{\ell=1}^{r}\left(\frac{b_{n}^{p}}{n} L_{n, I_{\ell}, t_{\ell}}\right)=\mu^{p}(f)^{r} \int_{(\mathbf{0}, \boldsymbol{t})} \prod_{i=1}^{K} h_{|\mathcal{I}(i)|}^{(\beta)}\left(\boldsymbol{x}_{\mathcal{I}(i)}\right) d \boldsymbol{x}
$$

where $(\mathbf{0}, \boldsymbol{t})=\left(0, t_{1}\right) \times \ldots \times\left(0, t_{r}\right), \boldsymbol{x}_{\mathcal{I}(i)}$ is the subvector of $\boldsymbol{x}:=\left(x_{1}, \ldots, x_{r}\right)$ indexed by $\mathcal{I}(i)$ (because each $h_{q}^{(\beta)}$ is symmetric, the order of variables in $\boldsymbol{x}_{\mathcal{I}(i)}$ does not matter $), d \boldsymbol{x}=d x_{1} \ldots d x_{r}$, and $K=\max \left(\bigcup_{\ell=1}^{r} I_{\ell}\right)$.

We are now ready to state the main reduction lemma.

Lemma 4.3. Suppose the convergence of finite-dimensional distributions in Theorem 3.2 or 3.3 hold for any symmetric Lévy measure $\rho=\rho_{0}$ so that (recall (18))

$$
\int_{\mathbb{R}} x^{r} \rho_{0}(d x)<\infty \text { for any } r \geq 0, \text { and } \int_{\mathbb{R}} x^{2} \rho_{0}(d x)=\left\|\rho_{0}^{\leftarrow}\right\|_{L^{2}\left(\mathbb{R}_{+}\right)}^{2}=1
$$

Then the corresponding convergence of finite-dimensional distributions also hold for general $\rho$ satisfying Assumption 圆. 
Proof. Fix the Lévy measure $\rho$ as in Assumption2. For any $\epsilon \in(0,1)$, there exists a symmetric Lévy measure $\rho_{0}=\rho_{0}^{(\epsilon)}$ satisfying (39) such that (recall the generalized inverse in (17))

$$
\left\|\rho^{\leftarrow}-\rho_{0}^{\leftarrow}\right\|_{L^{2}\left(\mathbb{R}_{+}\right)} \leq \epsilon
$$

Indeed, it is not difficult to construct the desired $\rho_{0}^{\overleftarrow{ }}$ as a right-continuous non-increasing simple function with a bounded support. Define

$$
W_{n, j}(t):=\sum_{k=1}^{\lfloor n t\rfloor} X_{k, j}^{(n)}, \quad j=2,3, \quad t \in[0,1],
$$

where $X_{k, 2}^{(n)}$ and $X_{k, 3}^{(n)}$ are as introduced in (31) corresponding to $\rho_{0}$ and $\rho$ respectively. Recall from (34) that $\left(X_{k, 3}^{(n)}, k=1, \ldots, n\right) \stackrel{d}{=}\left(X_{k}, k=1, \ldots, n\right)$, the latter being the stationary sequence in Theorems 3.2 or 3.3. On the other hand, $\left(X_{k, 2}^{(n)}, k=1, \ldots, n\right) \stackrel{d}{=}\left(X_{k}^{(\epsilon)}, k=1, \ldots, n\right)$, the latter being a stationary sequence defined using a multiple stochastic integral as $\left(X_{k}, k=1, \ldots, n\right)$, but with the only difference that the Lévy measure $\rho$ of the random measure $M$ is replaced by $\rho_{0}$. Then using the orthogonality induced by $\prod_{i \in I} \epsilon_{i}$ and independence, we have

$$
\begin{aligned}
\mathbb{E}\left|W_{n, 2}(t)-W_{n, 3}(t)\right|^{2} & =(p !)^{2} \mathbb{E}\left|\sum_{I \in \mathcal{D}_{p}}\left(\prod_{i \in I} \epsilon_{i}\right)\left(\prod_{i \in I} \rho_{0}^{\leftarrow}\left(\Gamma_{i} / w_{n}\right)-\prod_{i \in I} \rho^{\leftarrow}\left(\Gamma_{i} / w_{n}\right)\right)\left(\sum_{k=1}^{\lfloor n t\rfloor}\left(f \circ T_{p}^{k}\right)\left(U_{I, n}\right)\right)\right|^{2} \\
& =(p !)^{2}\left(\mathbb{E} \sum_{I \in \mathcal{D}_{p}}\left|\prod_{i \in I} \rho_{0}^{\leftarrow}\left(\Gamma_{i} / w_{n}\right)-\prod_{i \in I} \rho^{\leftarrow}\left(\Gamma_{i} / w_{n}\right)\right|^{2}\right) \mathbb{E} L_{n, I_{0}, t}^{2}
\end{aligned}
$$

where $I_{0}$ is an arbitrary fixed element of $\mathcal{D}_{p}$ and $L_{n, I_{0}, t}$ is as in (35). Set a sequence of $p$-variate functions as

$$
g_{q}\left(x_{1}, \ldots, x_{p}\right)=\prod_{i=1}^{q} \rho^{\leftarrow}\left(x_{i}\right) \prod_{i=q+1}^{p} \rho_{0}^{\leftarrow}\left(x_{i}\right), \quad q=0, \ldots, p,
$$

where a product is understood as 1 if the starting index exceeds the ending index. Note that the sum in the first expectation in (41) can be viewed as $\xi^{p}(h) / p !$, where $\xi^{p}(h):=\int_{\mathbb{R}_{+}^{p}}^{\prime} h\left(x_{1}, \ldots, x_{p}\right) \xi\left(d x_{1}\right) \ldots \xi\left(d x_{p}\right)$ is an offdiagonal multiple integral on $\mathbb{R}_{+}^{p}$ of the function $h:=\left|g_{0}-g_{p}\right|^{2}$ with respect to the Poisson random measure $\xi:=\sum_{i=1}^{\infty} \delta_{\Gamma_{i}}$. According to [16, Lemma 10.1(i)], the expectation $\mathbb{E} \xi^{p}(h)=\int_{\mathbb{R}_{+}^{p}} h\left(x_{1}, \ldots, x_{p}\right) d x_{1} \ldots d x_{p}$. Applying this with triangular inequalities, (40) and the fact $\epsilon \leq 1$, we have

$$
\begin{aligned}
& p ! \mathbb{E} \sum_{I \in \mathcal{D}_{p}}\left|\prod_{i \in I} \rho_{0}^{\leftarrow}\left(\Gamma_{i} / w_{n}\right)-\prod_{i \in I} \rho^{\leftarrow}\left(\Gamma_{i} / w_{n}\right)\right|^{2}=w_{n}^{p}\left\|g_{0}-g_{p}\right\|_{L^{2}\left(\mathbb{R}_{+}^{p}\right)} \leq w_{n}^{p}\left(\sum_{q=0}^{p-1}\left\|g_{q}-g_{q+1}\right\|_{L^{2}\left(\mathbb{R}_{+}^{p}\right)}\right)^{2} \\
& \leq w_{n}^{p}\left(p \max \left(\left\|\rho_{0}^{\leftarrow}\right\|_{L^{2}\left(\mathbb{R}_{+}\right)},\left\|\rho^{\leftarrow}\right\|_{L^{2}\left(\mathbb{R}_{+}\right)}\right)^{p-1}\left\|\rho^{\leftarrow}-\rho_{0}^{\leftarrow}\right\|_{L^{2}\left(\mathbb{R}_{+}\right)}\right)^{2} \leq w_{n}^{p} p^{2}\left(\left\|\rho^{\leftarrow}\right\|_{L^{2}\left(\mathbb{R}_{+}\right)}+1\right)^{2 p-2} \epsilon^{2} .
\end{aligned}
$$

On the other hand, by Lemmas 4.14 .2 and (12), there exists a constant $c>0$ which does not depend on $n$ or $\epsilon$ that

$$
\mathbb{E} L_{n, I_{0}, t}^{2} \leq \begin{cases}c n w_{n}^{-p} & \text { if } \beta<1-1 / p \\ c n^{2} w_{n}^{-2 p} & \text { if } \beta>1-1 / p .\end{cases}
$$

Hence returning to (41), for some constant $c>0$ which does not depend on $n, \epsilon$ or $\rho_{0}$, we have

$$
\mathbb{E}\left|W_{n, 2}(t)-W_{n, 3}(t)\right|^{2} \leq \begin{cases}c n \epsilon^{2} & \text { if } \beta<1-1 / p, \\ c n^{2} w_{n}^{-p} \epsilon^{2} & \text { if } \beta>1-1 / p\end{cases}
$$


Hence if $\beta<1-1 / p$, we have

$$
\mathbb{E}\left|n^{-1 / 2} W_{n, 2}(t)-n^{-1 / 2} W_{n, 3}(t)\right|^{2} \leq c \epsilon^{2} .
$$

Now by assumption

$$
\left(n^{-1 / 2} W_{n, 3}(t)\right) \stackrel{\text { f.d.d. }}{\longrightarrow}(\sigma B(t))
$$

where $B(t)$ is a standard Brownian motion and

$$
\sigma^{2}=\sum_{k=-\infty}^{\infty} \mathbb{E}\left[X_{k} X_{0}\right]=\sum_{k=-\infty}^{\infty} \mathbb{E}\left[X_{k}^{(\epsilon)} X_{0}^{(\epsilon)}\right]
$$

Note that here $\mathbb{E}\left[X_{k} X_{0}\right]=\mathbb{E}\left[X_{k}^{(\epsilon)} X_{0}^{(\epsilon)}\right]$. This is because although $\rho_{0}$ can be different from $\rho$, the same $L^{2}$ isometry relation (19) holds for both since they are standardized (see (15) and (39)). Hence the conclusion for the case $\beta<1-1 / p$ follows from a well-known approximation argument (e.g., [15, Theorem 4.28]). The case $\beta>1-1 / p$ is similar with the normalization $n^{1 / 2}$ in (42) replaced by $a_{n}$ in (22). Note that $a_{n} \sim c n w_{n}^{-p}$ in view of (12) as $n \rightarrow \infty$

In view of Lemma 4.3 and (33), it suffices to prove the convergences of finite-dimensional distributions in Theorems 3.2 and 3.3 with $\left(X_{k}\right)$ replaced by $\left(X_{k, 1}\right)$, the latter being defined by a compound Poisson random measure with all moments finite. This will be the objective of Sections 4.2 and 4.3 below.

\subsection{Proof of the central limit theorem}

Assume $p(\beta-1)<-1$. Using $\left(X_{k, 1}^{(n)}\right)_{1 \leq k \leq n}$ in (29), we define in this subsection

$$
\left(S_{n}(t)\right)_{t \in[0,1]}:=\left(\frac{1}{\sqrt{n}} \sum_{k=1}^{\lfloor n t\rfloor} X_{k, 1}^{(n)}\right)_{t \in[0,1]}=\left(p ! n^{-1 / 2} \sum_{I \in \mathcal{D}_{p}\left(N_{n}\right)}\left(\prod_{i \in I} Z_{i}\right) L_{n, I, t}\right)_{t \in[0,1]}
$$

where $N_{n}=N\left(Q w_{n}\right)$ and

$$
L_{n, I, t}=\sum_{k=1}^{\lfloor n t\rfloor}\left(f \circ T_{p}^{k}\right)\left(U_{I, n}\right)
$$

We need the following lemma when employing the method of moments.

Lemma 4.4. Under the assumptions in Section 3, for any $q \geq 2$ and any $b \in(\beta-1,0)$, there exists a constant $c>0$ which does not depend on $k_{1}, \ldots, k_{q}$, such that

$$
\mu\left(\bigcap_{j=1}^{q} T^{-k_{j}} A\right) \leq c\left(k_{2}-k_{1}\right)_{1}^{b}\left(k_{3}-k_{2}\right)_{1}^{b} \ldots\left(k_{q}-k_{q-1}\right)_{1}^{b}
$$

for all $1 \leq k_{1} \leq \ldots \leq k_{q}$, where $(x)_{1}^{b}:=(x \vee 1)^{b}$.

Proof. We shall prove the conclusion by induction. For $q=2$, using the measure-preserving property of $T$, we have

$$
\mu\left(T^{-k_{1}} A \cap T^{-k_{2}} A\right)=\mu\left(A \cap T^{-\left(k_{2}-k_{1}\right)} A\right) .
$$

Recall that by Potter's bound for regular variation [5, Theorem 1.5.6(i)], if a sequence $\left(a_{n}\right) \in \operatorname{RV}_{\infty}(-\gamma)$, $\gamma>0$, then for any $\gamma^{*} \in(0, \gamma)$, there exists some constant $c>0$ such that $a_{n} \leq c n^{-\gamma^{*}}$. Hence the conclusion follows from (13) and Potter's bound. Now suppose that the conclusion holds for $q \geq 2$, and we shall prove 
that it also holds for $q+1$. Indeed, using the measure preserving property of $T$ and the dual operator property (7),

$$
\begin{aligned}
\mu\left(\bigcap_{j=1}^{q+1} T^{-k_{j}} A\right) & =\mu\left(\bigcap_{j=1}^{q+1} T^{-\left(k_{j}-k_{1}\right)} A\right)=\int 1_{A} \times\left(1_{A} \times 1_{A} \circ T^{k_{3}-k_{2}} \times \ldots \times 1_{A} \circ T^{k_{q+1}-k_{2}}\right) \circ T^{k_{2}-k_{1}} d \mu \\
& =\int_{A}\left(\widehat{T}^{k_{2}-k_{1}} 1_{A}\right) \times\left(1_{A} \times 1_{A} \circ T^{k_{3}-k_{2}} \times \ldots \times 1_{A} \circ T^{k_{q+1}-k_{2}}\right) d \mu \\
& \leq c \mu(A)\left(k_{2}-k_{1}\right)_{1}^{b} \mu\left(\bigcap_{j=2}^{q+1} T^{-k_{j}} A\right)
\end{aligned}
$$

for some constant $c>0$, where for the inequality we have used (10) (note that $A$ is the whole subspace and thus $\mu$-a.e. continuous) and Potter's bound. Then the conclusion follows from the induction hypothesis.

Now we are ready to carry out the method of moments computation.

Proposition 4.5. Let $S_{n}(t)$ be as in (43) where $\rho_{0}$ defining $\left(X_{k, 1}^{(n)}\right)_{1 \leq k \leq n}$ satisfies the assumptions in Lemma 4.3. Assume $\beta<1-1 / p$. Then as $n \rightarrow \infty$,

$$
\mathbb{E}\left[S_{n}\left(t_{1}\right) \ldots S_{n}\left(t_{r}\right)\right] \rightarrow \sigma^{r} \mathbb{E}\left[B\left(t_{1}\right) \ldots B\left(t_{r}\right)\right]=\sigma^{r} \sum_{\mathcal{P}(r)} \prod_{j=1}^{r / 2}\left(t_{u_{j}} \wedge t_{v_{j}}\right)
$$

where

$$
\sigma^{2}:=\sum_{k=-\infty}^{\infty} \mathbb{E}\left[X_{k} X_{0}\right]=p ! \sum_{k=-\infty}^{\infty} \mu^{p}\left(\left(f \circ T_{p}^{k}\right) \cdot f\right)
$$

$B(t)$ is the standard Brownian motion, and $\mathcal{P}(r)$ denotes the collection of all the partitions of $\{1, \ldots, r\}$ into disjoint pairs $\left\{u_{j}, v_{j}\right\}, j=1, \ldots, r / 2$ if $r$ is even, and is understood as $\emptyset$ (hence the last expression in (44) is zero) if $r$ is odd.

Proof. The equality in (44) follows from [13, Theorem 1.28] and the covariance structure of a Brownian motion. So it is left to prove the convergence in (44).

We use the following notation throughout to denote conditional expectation given the Poisson process $N$ in (29):

$$
\mathbb{E}_{N}[\cdot]=\mathbb{E}[\cdot \mid N]
$$

If $r=1$, we have $\mathbb{E}\left[S_{n}\left(t_{1}\right)\right]=\mathbb{E}\left(\mathbb{E}_{N}\left[S_{n}\left(t_{1}\right)\right]\right)=0$ due to the symmetry of $Z_{i}$, and hence (44) holds. We assume $r \geq 2$ throughout below.

Part 1: The first part of the proof aims at showing as $n \rightarrow \infty$,

$$
\mathbb{E}_{N}\left[S_{n}\left(t_{1}\right) \ldots S_{n}\left(t_{r}\right)\right] \rightarrow \sigma^{r} \sum_{\mathcal{P}(r)} \prod_{j=1}^{r / 2}\left(t_{u_{j}} \wedge t_{v_{j}}\right) \quad \text { a.s.. }
$$

By independence, we have a.s.

$$
\mathbb{E}_{N}\left[S_{n}\left(t_{1}\right) \ldots S_{n}\left(t_{r}\right)\right]=(p !)^{r} n^{-r / 2} \sum_{I_{1}, \ldots, I_{r} \in \mathcal{D}_{p}\left(N_{n}\right)} \mathbb{E}\left[\left(\prod_{i \in I_{1}} Z_{i}\right) \ldots\left(\prod_{i \in I_{r}} Z_{i}\right)\right] \mathbb{E}\left(\prod_{\ell=1}^{r} L_{n, I_{\ell}, t_{\ell}}\right)
$$

Due to the symmetry of the distribution of $\left(Z_{i}\right)$, a factor $\mathbb{E}\left[\left(\prod_{i \in I_{1}} Z_{i}\right) \ldots\left(\prod_{i \in I_{r}} Z_{i}\right)\right] \neq 0$ if and only if the cardinality $|\mathcal{I}(i)|\left(\right.$ recall $\mathcal{I}(i)$ defined in (37)) is even for each $i=1, \ldots, N_{n}$, which can happen only if $p r$ is 


\begin{tabular}{|c|c|c|c|c|}
\hline$i$ & 1 & 2 & 3 & 4 \\
\hline$I_{1}$ & $\bullet$ & & & $\bullet$ \\
\hline$I_{2}$ & & $\bullet$ & $\bullet$ & \\
\hline$I_{3}$ & $\bullet$ & & & $\bullet$ \\
\hline$I_{4}$ & & $\bullet$ & $\bullet$ & \\
\hline
\end{tabular}

Figure 1: The configuration above corresponds to $p=2, r=4, I_{1}=I_{3}=\{1,4\}, I_{2}=I_{4}=\{2,3\}$. In this case, $\mathcal{I}(1)=\mathcal{I}(4)=\{1,3\}, \mathcal{I}(2)=\mathcal{I}(3)=\{2,4\}$. So $\left(I_{1}, I_{2}, I_{3}, I_{4}\right) \in \mathcal{C}(4) \cap \mathcal{N}(4)$.

even. If $p r$ is odd, then so is $r$, and hence the limit in (45) is zero. So 45) trivially holds when $p r$ is odd. We shall assume $p r$ is even below throughout the proof of Part 1.

We shall analyze (46) by decomposing it into contributing and negligible terms. For this purpose, we introduce for $m \geq p$,

$$
\mathcal{M}(m)=\left\{\left(I_{1}, \ldots, I_{r}\right): I_{\ell} \in \mathcal{D}_{p}(m), \ell=1, \ldots, r,|\mathcal{I}(i)| \text { is even for } i=1, \ldots, m\right\},
$$

where $\mathcal{D}_{p}(m)$ is as in (30). When $r$ is an even integer, we define

$$
\mathcal{C}(m)=\left\{\left(I_{1}, \ldots, I_{r}\right) \in \mathcal{M}(m) \text { : exactly } r / 2 \text { pairs of } I_{\ell} \text { 's coincide and different pairs are disjoint. }\right\} .
$$

When $r$ is odd, set $\mathcal{C}(m)=\emptyset$. Define for $m=p, p+1, \ldots, p r / 2$ that

$$
\mathcal{N}(m)=\left\{\left(I_{1}, \ldots, I_{r}\right) \in \mathcal{M}(m): \mathcal{I}(i) \neq \emptyset, i=1, \ldots, m\right\} .
$$

Note that we have suppressed in notation the dependence of $\mathcal{M}(m), \mathcal{C}(m)$ and $\mathcal{N}(m)$ on $p$ and $r$. See Figures 1 and 2 for illustrations of the notation introduced above.

Since $N_{n} \uparrow \infty$ as $n \rightarrow \infty$ a.s., we can assume without loss of generality that $N_{n} \geq p$. By the arguments below (46), the index set $\mathcal{D}_{p}\left(N_{n}\right)$ under the summation sign in (46) can be replaced by $\mathcal{M}\left(\mathcal{N}_{n}\right)$. Decompose the sum in (46) into

$$
A(n)=(p !)^{r} n^{-r / 2} \sum_{\left(I_{1}, \ldots, I_{r}\right) \in \mathcal{C}\left(N_{n}\right)} \mathbb{E}\left[\left(\prod_{i \in I_{1}} Z_{i}\right) \ldots\left(\prod_{i \in I_{r}} Z_{i}\right)\right] \mathbb{E}\left(\prod_{\ell=1}^{r} L_{n, I_{\ell}, t_{\ell}}\right)
$$

and

$$
B(n)=(p !)^{r} n^{-r / 2} \sum_{\left(I_{1}, \ldots, I_{r}\right) \in \mathcal{M}\left(N_{n}\right) \backslash \mathcal{C}\left(N_{n}\right)} \mathbb{E}\left[\left(\prod_{i \in I_{1}} Z_{i}\right) \ldots\left(\prod_{i \in I_{r}} Z_{i}\right)\right] \mathbb{E}\left(\prod_{\ell=1}^{r} L_{n, I_{\ell}, t_{\ell}}\right) .
$$

Note that both $A(n)$ and $B(n)$ are stochastic since they depend on the Poisson count $N_{n}$. We shall show that $A(n)$ is the contributing term while $B(n)$ is negligible.

We assume that $r$ is even so that $A(n)$ is possibly nonzero. To enumerate the elements in $\mathcal{C}\left(N_{n}\right)$, first select a partition from $\mathcal{P}(r)$ which specifies the pairings among $I_{1}, \ldots, I_{r}$. Then assign $\left(\begin{array}{c}N_{n} \\ p\end{array}\right)$ elements from $\left\{1, \ldots, N_{n}\right\}$ to the 1st pair, assign $\left(\begin{array}{c}N_{n}-p \\ p\end{array}\right)$ from the rest to the 2 nd pair, .., and assign $\left(\begin{array}{c}N_{n}-p r / 2 \\ p\end{array}\right)$ from the rest to the last pair. Therefore, using the fact that $Z_{i}$ follows the distribution $\rho_{0}(\cdot) / Q$ and the relation (39), we have

$$
A(n)=(p !)^{r} n^{-r / 2} \sum_{\mathcal{P}(r)}\left(\begin{array}{c}
N_{n} \\
p
\end{array}\right)\left(\begin{array}{c}
N_{n}-p \\
p
\end{array}\right) \ldots\left(\begin{array}{c}
N_{n}-p r / 2 \\
p
\end{array}\right) Q^{-p r / 2} \prod_{j=1}^{r / 2} \mathbb{E}\left[L_{n, I, t_{u_{j}}} L_{n, I, t_{v_{j}}}\right]
$$

where the sum is over partitions $\left\{\left\{u_{j}, v_{j}\right\}, j=1, \ldots, r / 2\right\} \in \mathcal{P}(r)$. Applying Lemma 4.1 and the fact $N_{n} \sim Q w_{n}$ a.s., we have a.s.

$$
A(n) \sim n^{-r / 2} \sum_{\mathcal{P}(r)}\left(Q w_{n}\right)^{p r / 2} Q^{-p r / 2} \prod_{j=1}^{r / 2}\left(\left(\sum_{k=-\infty}^{\infty} \mu^{p}\left(\left(f \circ T_{p}^{k}\right) f\right)\right)\left(t_{u_{j}} \wedge t_{v_{j}}\right) n w_{n}^{-p}\right),
$$


which simplifies to the right-hand side of (45).

Next we show that $B(n) \rightarrow 0$ a.s. as $n \rightarrow \infty$. First by Hölder's inequality,

$$
\left|\mathbb{E}\left[\left(\prod_{i \in I_{1}} Z_{i}\right) \ldots\left(\prod_{i \in I_{r}} Z_{i}\right)\right]\right| \leq \mathbb{E}\left|Z_{1}\right|^{p r}<\infty .
$$

Second, the joint law of $\left(L_{n, I_{\ell}, t_{\ell}}, \ell=1, \ldots, r\right)$ is unchanged if the elements in $I_{\ell}, \ell=1, \ldots, r$, are replaced by the elements of $\{1, \ldots, m\}, m=\left|\cup_{\ell=1}^{r} I_{\ell}\right|$, based on any one-to-one correspondence. Exploring these facts we have

$$
|B(n)| \leq c n^{-r / 2} \sum_{m=p}^{p r / 2}\left(\begin{array}{c}
N_{n} \\
m
\end{array}\right) \sum_{\left(I_{1}, \ldots, I_{r}\right) \in \mathcal{N}(m) \backslash \mathcal{C}(m)} \mathbb{E}\left(\prod_{\ell=1}^{r}\left|L_{n, I_{\ell}, t_{\ell}}\right|\right) .
$$

Fix for now $m$ and $\left(I_{1}, \ldots, I_{r}\right) \in \mathcal{N}(m) \backslash \mathcal{C}(m)$. Our next goal is to provide a bound for $\mathbb{E}\left(\prod_{\ell=1}^{r}\left|L_{n, I_{\ell}, t_{\ell}}\right|\right)$. It follows from a triangular inequality, the restriction $t_{\ell} \in[0,1]$ and the assumptions on $f$ that

$$
\left|L_{n, I_{\ell}, t_{\ell}}\right| \leq c \sum_{k=1}^{n}\left(1_{A^{p}} \circ T_{p}^{k}\right)\left(U_{I_{\ell}, n}\right) .
$$

Hence

$$
\mathbb{E}\left(\prod_{\ell=1}^{r}\left|L_{n, I_{\ell}, t_{\ell}}\right|\right) \leq c \sum_{k_{1}, \ldots, k_{r}=1}^{n} \prod_{i=1}^{m} \int\left(\prod_{\ell \in \mathcal{I}(i)} 1_{A} \circ T^{k_{l}}\right) d \mu_{n}=c \sum_{k_{1}, \ldots, k_{r}=1}^{n} \prod_{i=1}^{m} f_{|\mathcal{I}(i)|, n}\left(k_{\mathcal{I}(i)}\right),
$$

where $\mathcal{I}(i)$ is as in (37) and

$$
f_{q, n}:\{1, \ldots, n\}^{q} \rightarrow[0,1], \quad f_{q, n}\left(k_{1}, \ldots, k_{q}\right)= \begin{cases}1 & \text { if } q=0 \\ \mu\left(\bigcap_{\ell=1}^{q} T^{-k_{\ell}} A\right) / w_{n} & \text { if } q \geq 1 .\end{cases}
$$

Note that since $f_{q, n}$ is symmetric, the order of the variables in $k_{\mathcal{I}(i)}$ does not matter. By Lemma 4.4 and the measure-preserving property of $T$, we have

$$
f_{q, n}\left(k_{1}, \ldots, k_{q}\right) \leq c \begin{cases}1 & \text { if } q=0 \\ w_{n}^{-1} & \text { if } q=1 \\ w_{n}^{-1}\left(k_{2}-k_{1}\right)_{1}^{b} \ldots\left(k_{q}-k_{q-1}\right)_{1}^{b} & \text { if } q \geq 2\end{cases}
$$

where $b$ is chosen to satisfy (recall $\beta-1<-1 / p$ )

$$
(\beta-1) \vee\left(-\frac{1}{p-1}\right)<b<-\frac{1}{p} .
$$

Next, we shall provide a bound for

$$
\sum_{1 \leq k_{1} \leq \ldots \leq k_{r} \leq n} \prod_{i=1}^{m} f_{|\mathcal{I}(i)|, n}\left(k_{\mathcal{I}(i)}\right),
$$

which in turn yields a bound for the full sum in (55) by adding up all $r$ ! orders of $k_{1}, \ldots, k_{r}$. Suppose $\mathcal{I}(i)=\{u(i, 1), u(i, 2) \ldots, u(i,|\mathcal{I}(i)|)\} \subset\{1, \ldots, r\}, u(i, 1)<\ldots<u(i,|\mathcal{I}(i)|), i=1, \ldots, m$. When $1 \leq k_{1} \leq$ $\ldots \leq k_{r} \leq n$, one has

$$
\prod_{i=1}^{m} f_{|\mathcal{I}(i)|, n}\left(k_{\mathcal{I}(i)}\right)=w_{n}^{-m} \prod_{i=1}^{m} \prod_{s=2}^{|\mathcal{I}(i)|}\left(k_{u(i, s)}-k_{u(i, s-1)}\right)_{1}^{b} \leq w_{n}^{-m} \prod_{i=1}^{m} \prod_{s=2}^{|\mathcal{I}(i)|}\left(k_{u(i, s)}-k_{u(i, s)-1}\right)_{1}^{b},
$$




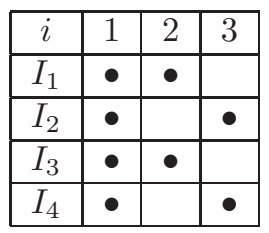

Figure 2: The configuration above corresponds to $p=2, r=4, m=3, I_{1}=I_{3}=\{1,2\}, I_{2}=I_{4}=\{1,3\}$. So $\left(I_{1}, I_{2}, I_{3}, I_{4}\right) \in \mathcal{N}(3) \backslash \mathcal{C}(3)$ and $\mathcal{I}(1)=\{1,2,3,4\}, \mathcal{I}(2)=\{1,3\}, \mathcal{I}(3)=\{2,4\}$. When $1 \leq k_{1} \leq k_{2} \leq$ $k_{3} \leq k_{4} \leq n$, we have $w_{n}^{3} \prod_{i=1}^{3} f_{|\mathcal{I}(i)|, n}\left(k_{\mathcal{I}(i)}\right)=\left(k_{2}-k_{1}\right)_{1}^{b}\left(k_{3}-k_{2}\right)_{1}^{b}\left(k_{4}-k_{3}\right)_{1}^{b} \times\left(k_{3}-k_{1}\right)_{1}^{b} \times\left(k_{4}-k_{2}\right)_{1}^{b} \leq$ $\left(k_{2}-k_{1}\right)_{1}^{b}\left(k_{3}-k_{2}\right)_{1}^{2 b}\left(k_{4}-k_{3}\right)_{1}^{2 b}$.

where the product $\prod_{s=2}^{|\mathcal{I}(i)|}$ is understood as 1 if $|\mathcal{I}(i)|<2$, and for the replacement of $u(i, s-1)$ by $u(i, s)-1$ in the inequality, we have used the monotonicity of the function $x \mapsto(x)_{1}^{b}$. Next, by rearranging the product, we have

$$
\prod_{i=1}^{m} \prod_{s=2}^{|\mathcal{I}(i)|}\left(k_{u(i, s)}-k_{u(i, s)-1}\right)_{1}^{b}=\prod_{u=2}^{r}\left(k_{u}-k_{u-1}\right)_{1}^{d_{u} b}
$$

where

$$
d_{u}=\mid\left\{1 \leq i \leq m: u \in \mathcal{I}(i), \exists u^{\prime} \in \mathcal{I}(i) \text { s.t. } u^{\prime}<u\right\} \mid, \quad u=2, \ldots, r .
$$

See Figure2 for an illustration of the bound of $\prod_{i=1}^{m} f_{|\mathcal{I}(i)|, n}\left(k_{\mathcal{I}(i)}\right)$ above. Let $L=\left|\left\{2 \leq u \leq r: d_{u}<p\right\}\right|+1$. Then

$$
m=\left|I_{1}\right|+\left|I_{2} \backslash I_{1}\right|+\ldots+\left|I_{r} \backslash\left(\cup_{j=1}^{r-1} I_{j}\right)\right|=p+\left(p-d_{2}\right)+\ldots+\left(p-d_{r}\right)=p L-\sum_{u=2}^{r} d_{u} 1_{\left\{d_{u}<p\right\}} .
$$

By (56), we have $d_{u} b<-1$ if $d_{u}=p$ and $d_{u} b>-1$ if $d_{u}<p$. So summing iteratively in the order $k_{r}, k_{r-1}, \ldots, k_{1}$, we obtain

$$
\sum_{1 \leq k_{1} \leq \ldots \leq k_{r} \leq n} \prod_{u=2}^{r}\left(k_{u}-k_{u-1}\right)_{1}^{d_{u} b} \leq c n^{\sum_{u=2}^{r}\left(1+d_{u} b\right) 1_{\left\{d_{u}<p\right\}}+1}=c n^{(p b+1) L-b m},
$$

where for the last equality we have used the relation (57) and the definition of $L$. There are two cases: (i) $\sum_{u=2}^{p} d_{u} 1_{\left\{d_{u}<p\right\}}>0$ and (ii) $\sum_{u=2}^{p} d_{u} 1_{\left\{d_{u}<p\right\}}=0$. In case (i), from (57) we deduce $L \geq(m+1) / p$. Hence the exponent in the bound (58) satisfies

$$
(p b+1) L-b m \leq(p b+1)(m+1) / p-b m=\frac{m}{p}+b+1 / p<\frac{m}{p} \leq \frac{r}{2},
$$

where the last inequality holds since $m \leq p r / 2$. In case (ii), since each $d_{u}=0$ or $p$, the index sets $I_{\ell}$ 's either coincide or disjoint. Because also $\left(I_{1}, \ldots, I_{r}\right) \in \mathcal{N}(m) \backslash \mathcal{C}(m)$, it is not difficult to see that $m \leq p r / 2-p$. Note also $L \geq m / p$ in view of (57). Hence

$$
(p b+1) L-b m \leq(p b+1) m / p-b m=\frac{m}{p} \leq \frac{r}{2}-1<\frac{r}{2} .
$$

Combining these two cases and returning to (55), we conclude that for some constant $\delta>0$ which does not depend on $n$ or $m$,

$$
\mathbb{E}\left(\prod_{\ell=1}^{r}\left|L_{n, I_{\ell}, t_{\ell}}\right|\right) \leq c n^{r / 2-\delta} w_{n}^{-m}
$$

for any $\left(I_{1}, \ldots, I_{r}\right) \in \mathcal{N}(m) \backslash \mathcal{C}(m)$. Plugging (59) into (53) and using the fact that $N_{n} \sim Q w_{n}$ a.s. as $n \rightarrow \infty$, we have $B(n) \rightarrow 0$ a.s.. 
Part 2. The second part of the proofs aims at establishing the uniform integrability of

$$
\left\{\mathbb{E}_{N}\left[S_{n}\left(t_{1}\right) \ldots S_{n}\left(t_{r}\right)\right], n \geq 1\right\}
$$

which combined with (45) concludes the proof. For this it suffices to show the uniform boundedness in $n$ of

$$
\mathbb{E}\left[\mathbb{E}_{N}\left[S_{n}\left(t_{1}\right) \ldots S_{n}\left(t_{r}\right)\right]^{2}\right] \leq \mathbb{E}\left[\mathbb{E}_{N}\left[S_{n}\left(t_{1}\right)^{2} \ldots S_{n}\left(t_{r}\right)^{2}\right]\right]
$$

Similarly as the way (53) is obtained, we have

$$
\mathbb{E}_{N}\left[S_{n}\left(t_{1}\right)^{2} \ldots S_{n}\left(t_{r}\right)^{2}\right] \leq c \sum_{m=p}^{p r}\left(\begin{array}{c}
N_{n} \\
m
\end{array}\right) \frac{1}{n^{r}} \sum_{\left(I_{1}, \ldots, I_{2 r}\right) \in \mathcal{N}(m)} \mathbb{E}\left(\prod_{\ell=1}^{2 r}\left|L_{n, I_{\ell}, t_{\ell}}\right|\right),
$$

where $\mathcal{N}(m)$ is as in (49) but with $r$ replaced by $2 r$. We claim for each $\left(I_{1}, \ldots, I_{2 r}\right) \in \mathcal{N}(m)$ that

$$
\mathbb{E}\left(\prod_{\ell=1}^{2 r}\left|L_{n, I_{\ell}, t_{\ell}}\right|\right) \leq c w_{n}^{-m} n^{r}
$$

Indeed, starting with the bound (54), this follows from arguments similar to Part 1: introducing $\mathcal{C}(m)$ as (48) but with $r$ replaced by $2 r$, dividing $\mathcal{N}(m)$ into $\mathcal{N}(m) \cap \mathcal{C}(m)$ and $\mathcal{N}(m) \backslash \mathcal{C}(m)$ (note $\mathcal{C}(m)$ is nonempty only when $m=p r$ ), and then applying Lemma 4.1 and (59) respectively in these two cases. So

$$
\mathbb{E}_{N}\left[S_{n}\left(t_{1}\right)^{2} \ldots S_{n}\left(t_{r}\right)^{2}\right] \leq c \sum_{m=p}^{p r} \frac{N_{n}\left(N_{n}-1\right) \ldots\left(N_{n}-m+1\right)}{w_{n}^{m}}
$$

Applying the fact $\mathbb{E}\left[N_{n}\left(N_{n}-1\right) \ldots\left(N_{n}-m+1\right)\right]=Q^{m} w_{n}^{m}$ concludes the desired boundedness.

Proof of Theorem 3.2. Recall for a random vector $\left(\xi_{1}, \ldots, \xi_{d}\right), d \in \mathbb{Z}_{+}$, the set of its (multivariate) moments is $\left\{E\left[\xi_{1}^{m_{1}} \ldots \xi_{d}^{m_{d}}\right]: m_{1}, \ldots, m_{d} \in\{0,1,2, \ldots\}\right\}$ given that all the expectations exist. The distribution of $\left(\xi_{1}, \ldots, \xi_{d}\right)$ is said to be moment-determinate if it is the only distribution with such a set of moments. It is known (see, e.g., 31, Theorem 14.6]) that the moment determinancy of every marginal univariate distribution of $\xi_{i}, i=1, \ldots, d$, implies the moment determinancy of the joint distribution of $\left(\xi_{1}, \ldots, \xi_{d}\right)$. In addition, it is well-known that if a limit distribution (univariate or multivariate) is moment-determinate, then the convergence of all the moments implies the weak convergence to the limit distribution. Suppose first that $\rho=\rho_{0}$ as in Lemma 4.3. Focusing on the joint distributions at a fixed finite set of time points, the convergence of all the moments follows from Proposition 4.5 (note that the time points $t_{1}, \ldots, t_{r}$ in Proposition 4.5 are allowed to coincide.). It is well-known that a univariate Gaussian distribution is moment-determinate, and hence so is a multivariate Gaussian distribution. So the convergence of the finite-dimensional distributions holds in this case. The extension from $\rho_{0}$ to general $\rho$ follows from Lemma 4.3 .

We are left to show the tightness in $D[0,1]$ with the uniform metric under the additional condition $\int_{\mathbb{R}} x^{4} \rho(d x)<\infty$, which by $[18$ is equivalent to

$$
\int_{0}^{\infty} \rho^{\leftarrow}(x)^{4} d x<\infty
$$

Define

$$
S_{n}^{\prime}(t)=\frac{1}{\sqrt{n}} \sum_{k=1}^{\lfloor n t\rfloor} X_{k, 3}^{(n)}=p ! \sum_{I \in \mathcal{D}_{p}}\left(\prod_{i \in I} \epsilon_{i} \rho^{\leftarrow}\left(\Gamma_{i} / w_{n}\right)\right)\left(\frac{1}{\sqrt{n}} \sum_{k=1}^{\lfloor n t\rfloor}\left(f \circ T_{p}^{k}\right)\left(U_{I, n}\right)\right), \quad t \in[0,1],
$$


which, in view of (34), has the same finite-dimensional distributions with the process defined as above but with $\left(X_{k, 3}^{(n)}\right)_{1 \leq k \leq n}$ replaced by $\left(X_{k}\right)_{1 \leq k \leq n}$. For $0 \leq t_{1}<t_{2} \leq 1$ and $I \in \mathcal{D}_{p}$, define the measurable map

$$
L_{n, t_{1}, t_{2}}: A_{n}^{p} \rightarrow \mathbb{R}, \quad L_{n, t_{1}, t_{2}}\left(u_{1}, \ldots, u_{p}\right):=\frac{1}{\sqrt{n}} \sum_{k=1}^{\left\lfloor n t_{2}\right\rfloor-\left\lfloor n t_{1}\right\rfloor}\left(f \circ T_{p}^{k}\right)\left(u_{1}, \ldots, u_{p}\right) .
$$

Using the stationarity of $\left(X_{k, 3}^{(n)}\right)_{1 \leq k \leq n}$ and applying a generalized Khinchine inequality for multilinear forms in Rademacher random variables (29, Theorem 1.3 (ii)]) conditioning on $\left(\Gamma_{i}\right)$ and $\left(U_{i, n}\right)$, we have

$$
\begin{aligned}
\mathbb{E}\left[S_{n}^{\prime}\left(t_{2}\right)-S_{n}^{\prime}\left(t_{1}\right)\right]^{4} & =\mathbb{E}\left[\left(p ! \sum_{I \in \mathcal{D}_{p}}\left(\prod_{i \in I} \epsilon_{i} \rho^{\leftarrow}\left(\Gamma_{i} / w_{n}\right)\right) L_{n, t_{1}, t_{2}}\left(U_{I, n}\right)\right)^{4}\right] \\
& \leq c \mathbb{E}\left[\left(\sum_{I \in \mathcal{D}_{p}}\left(\prod_{i \in I} \rho^{\leftarrow}\left(\Gamma_{i} / w_{n}\right)^{2}\right) L_{n, t_{1}, t_{2}}\left(U_{I, n}\right)^{2}\right)^{2}\right] .
\end{aligned}
$$

The sum inside the square in (62) can be viewed as an off-diagonal multiple integral of the integrand

$$
f_{n}\left(x_{1}, u_{1}, \ldots, x_{p}, u_{p}\right)=\left(\prod_{i=1}^{p} \rho^{\leftarrow}\left(x_{i} / w_{n}\right)^{2}\right) L_{n, t_{1}, t_{2}}\left(u_{1}, \ldots, u_{p}\right)^{2}
$$

on $\left(\mathbb{R}_{+} \times A_{n}\right)^{p}$ with respect to the (marked) Poisson random measure $\sum_{i=1}^{\infty} \delta_{\Gamma_{i}, U_{i, n}}$ with intensity measure $\nu_{n}:=\lambda \times \mu_{n}$ (e.g., [15, Lemma 12.2]), where $\lambda$ is the Lebesgue measure and $\mu_{n}$ is as in (24). So by [16, Lemma 10.1(iii)],

$$
\mathbb{E}\left[S_{n}^{\prime}\left(t_{2}\right)-S_{n}^{\prime}\left(t_{1}\right)\right]^{4} \leq c \sum_{m=0}^{p} m !\left(\begin{array}{c}
p \\
m
\end{array}\right)^{2} \nu_{n}^{p-m}\left(\nu_{n}^{m}\left(f_{n}\right)^{2}\right),
$$

where $\nu_{n}^{m}\left(f_{n}\right)$ is understood as integrating out $m$ of the $p$ variables of the symmetric function $f_{n}\left(\nu_{n}^{0} f=f\right)$, and $\nu_{n}^{p-m}$ integrates out the $p-m$ variables left in $\nu_{n}^{m}\left(f_{n}\right)^{2}$. Next, it can be verified that

$$
\nu_{n}^{p-m}\left(\nu_{n}^{m}\left(f_{n}\right)^{2}\right)=w_{n}^{p+m}\left(\int_{0}^{\infty} \rho^{\leftarrow}(x)^{4} d x\right)^{p-m} \mathbb{E}\left[L_{n, t_{1}, t_{2}}\left(U_{I_{1}, n}\right)^{2} L_{n, t_{1}, t_{2}}\left(U_{I_{2}, n}\right)^{2}\right],
$$

where $I_{1}, I_{2}$ are arbitrary elements of $\mathcal{D}_{p}$ satisfying $\left|I_{1} \cap I_{2}\right|=p-m$ (so $\left|I_{1} \cup I_{2}\right|=p+m$ ). Similarly as how (60) is obtained, using Lemma 4.1 and a bound as in (59) with $n$ replaced by $\left\lfloor n t_{2}\right\rfloor-\left\lfloor n t_{1}\right\rfloor$, we have

$$
\mathbb{E}\left[L_{n, t_{1}, t_{2}}\left(U_{I_{1}, n}\right)^{2} L_{n, t_{1}, t_{2}}\left(U_{I_{2}, n}\right)^{2}\right] \leq c w_{n}^{-\left|I_{1} \cup I_{2}\right|}\left(\frac{\left\lfloor n t_{2}\right\rfloor-\left\lfloor n t_{1}\right\rfloor}{n}\right)^{2} .
$$

Combining these above we obtain

$$
\mathbb{E}\left[S_{n}^{\prime}\left(t_{2}\right)-S_{n}^{\prime}\left(t_{1}\right)\right]^{4} \leq c\left(\frac{\left\lfloor n t_{1}\right\rfloor-\left\lfloor n t_{2}\right\rfloor}{n}\right)^{2},
$$

which concludes tightness in $D[0,1]$ in view of $[9$, Lemma 4.4.1]. 


\subsection{Proof of the non-central limit theorem}

Now assume $p(\beta-1) \in(-1,0)$. Using $\left(X_{k, 1}^{(n)}\right)_{1 \leq k \leq n}$ in (29), we define in this subsection

$$
\left(S_{n}^{*}(t)\right)_{t \in[0,1]}=\left(\frac{b_{n}^{p}}{w_{n}^{p / 2} n} \sum_{k=1}^{\lfloor n t\rfloor} X_{k, 1}^{(n)}\right)_{t \in[0,1]} \stackrel{d}{=}\left(p ! w_{n}^{-p / 2} \sum_{I \in \mathcal{D}_{p}\left(N_{n}\right)}\left(\prod_{i \in I} Z_{i}\right) L_{n, I, t}^{*}\right)_{t \in[0,1]}
$$

where $N_{n}=N\left(Q w_{n}\right)$ and

$$
L_{n, I, t}^{*}=\frac{b_{n}^{p}}{n} \sum_{k=1}^{\lfloor n t\rfloor}\left(f \circ T_{p}^{k}\right)\left(U_{I, n}\right) .
$$

Note that $\left(\frac{b_{n}^{p}}{w_{n}^{p / 2} n}\right) \in \mathrm{RV}_{\infty}(p(1-\beta) / 2-1)$, where $p(1-\beta) / 2-1 \in(-1,-1 / 2)$.

Proposition 4.6. Let $S_{n}^{*}(t)$ be as in (63) where $\rho_{0}$ defining $\left(X_{k, 1}^{(n)}\right)_{1 \leq k \leq n}$ satisfies the assumptions in Lemma 4.3. Assume $\beta \in(1-1 / p, 1)$. Then as $n \rightarrow \infty$,

$$
\mathbb{E}\left[S_{n}^{*}\left(t_{1}\right) \ldots S_{n}^{*}\left(t_{r}\right)\right] \rightarrow \mathbb{E}\left[H_{p, \beta}\left(t_{1}\right) \ldots H_{p, \beta}\left(t_{r}\right)\right],
$$

where $H_{p, \beta}(t)$ is a constant multiple of the standard Hermite process $Z_{p, \beta}(t)$ in (3).

Proof. Let $E_{N}$ denote the conditional expectation given $N$. We have

$$
\mathbb{E}_{N}\left[S_{n}^{*}\left(t_{1}\right) \ldots S_{n}^{*}\left(t_{r}\right)\right]=(p !)^{r} w_{n}^{-r p / 2} \sum_{I_{1}, \ldots, I_{r} \in \mathcal{D}_{p}\left(N_{n}\right)} \mathbb{E}\left[\left(\prod_{i \in I_{1}} Z_{i}\right) \ldots\left(\prod_{i \in I_{r}} Z_{i}\right)\right] \mathbb{E}\left(\prod_{\ell=1}^{r} L_{n, I_{\ell}, t_{\ell}}^{*}\right) .
$$

Assume without loss of generality that $N_{n} \geq p r / 2$. Similarly as the arguments below (46), we can assume that $p r$ is even. Recalling $\mathcal{M}(m)$ in (47) and $\mathcal{N}(m)$ in (49), we have

$$
\begin{aligned}
\mathbb{E}_{N}\left[S_{n}^{*}\left(t_{1}\right) \ldots S_{n}^{*}\left(t_{r}\right)\right] & =(p !)^{r} w_{n}^{-p r / 2} \sum_{\left(I_{1}, \ldots, I_{r}\right) \in \mathcal{M}\left(N_{n}\right)} \mathbb{E}\left[\left(\prod_{i \in I_{1}} Z_{i}\right) \ldots\left(\prod_{i \in I_{r}} Z_{i}\right)\right] \mathbb{E}\left(\prod_{\ell=1}^{r} L_{n, I_{\ell}, t_{\ell}}^{*}\right) \\
& =\sum_{m=p}^{p r / 2}(p !)^{r} w_{n}^{-p r / 2}\left(\begin{array}{c}
N_{n} \\
m
\end{array}\right) \sum_{\left(I_{1}, \ldots, I_{r}\right) \in \mathcal{N}(m)} \mathbb{E}\left[\left(\prod_{i \in I_{1}} Z_{i}\right) \ldots\left(\prod_{i \in I_{r}} Z_{i}\right)\right] \mathbb{E}\left(\prod_{\ell=1}^{r} L_{n, I_{\ell}, t_{\ell}}^{*}\right) \\
& =: \sum_{m=p}^{p r / 2} T_{m}(n),
\end{aligned}
$$

where the second equality above follows from an argument similar to the one leading to (53).

We first show that

$$
\lim _{n} T_{m}(n)=0 \quad \text { for } m=p, \ldots, p r / 2-1 .
$$

For this purpose, note first that $\mathbb{E}\left[\prod_{\ell=1}^{r} L_{n, I_{\ell}, t_{\ell}}^{*}\right]$ are uniformly bounded with respect to $0 \leq t_{\ell} \leq 1$ and $I_{\ell}, \ell=1, \ldots, r$. Indeed, this can be seen by bounding $|f|$ with a constant multiple of $1_{A^{p}}$, replacing $t_{\ell}$ 's by 1 , and applying Lemma 4.2 with $f=1_{A^{p}}$. In addition, $\mathbb{E}\left(\left(\prod_{i \in I_{1}} Z_{i}\right) \ldots\left(\prod_{i \in I_{r}} Z_{i}\right)\right)$ are also uniformly bounded in view of (52). Therefore,

$$
T_{m}(n) \leq c w_{n}^{-p r / 2}\left(\begin{array}{c}
N_{n} \\
m
\end{array}\right), \quad m=p, \ldots, p r / 2 .
$$

The right-hand side of (67) tends to 0 a.s. if $m<p r / 2$ since $N_{n} / w_{n} \rightarrow Q$ a.s.. 
Now we treat the leading term $m=p r / 2$. Note that a configuration $\left(I_{1}, \ldots, I_{r}\right)$ belongs to $\mathcal{N}(p r / 2)$ if and only if $|\mathcal{I}(i)|=2$ (recall (37) and (49)) for all $i=1, \ldots, p r / 2$. Hence by Lemma 4.2 and (39),

$$
\begin{aligned}
T_{p r / 2}(n) & =(p !)^{r} w_{n}^{-p r / 2}\left(\begin{array}{c}
N_{n} \\
p r / 2
\end{array}\right) \sum_{\left(I_{1}, \ldots, I_{r}\right) \in \mathcal{N}(p r / 2)} \mathbb{E}\left[\left(\prod_{i \in I_{1}} Z_{i}\right) \ldots\left(\prod_{i \in I_{r}} Z_{i}\right)\right] \mathbb{E}\left(\prod_{\ell=1}^{r} L_{n, I_{\ell}, t_{\ell}}^{*}\right) \\
& \rightarrow \frac{(p !)^{r} Q^{p r / 2}}{(p r / 2) !} \mu^{p}(f)^{r} \sum_{\left(I_{1}, \ldots, I_{r}\right) \in \mathcal{N}(p r / 2)} \int_{(\mathbf{0}, \boldsymbol{t})} \prod_{i=1}^{p r / 2} h_{2}^{(\beta)}\left(\boldsymbol{x}_{\mathcal{I}(i)}\right) d \boldsymbol{x} \quad \text { a.s. },
\end{aligned}
$$

We claim that the summation above equals

$$
2^{-p r / 2}[\Gamma(\beta) \Gamma(2-\beta)]^{p r / 2} \sum \int_{0}^{t_{1}} d s_{1} \ldots \int_{0}^{t_{r}} d s_{r}\left|s_{u(1)}-s_{v(1)}\right|^{\beta-1} \ldots\left|s_{u(p r / 2)}-s_{v(p r / 2)}\right|^{\beta-1},
$$

where the sum above is over all indices $u(1), v(1), \ldots, u(\mathrm{pr} / 2), v(\mathrm{pr} / 2) \in\{1, \ldots, r\}$ such that $u(1) \neq v(1), \ldots$, $u(p r / 2) \neq v(p r / 2)$ and each number $1, \ldots, r$ appears exactly $p$ times in $u(1), v(1), \ldots, u(p r / 2), v(p r / 2)$. To see this, write $\mathcal{I}(i)=\{u(i), v(i)\}, i=1, \ldots, p r / 2$, and note that the factor $2^{-p r / 2}$ above accounts for the ignorance of the order within each pair $(u(i), v(i))$ in $\mathcal{I}(i)$.

Combining these above and returning to (66), we get as $n \rightarrow \infty$ that

$$
\begin{aligned}
& \mathbb{E}_{N}\left[S_{n}^{*}\left(t_{1}\right) \ldots S_{n}^{*}\left(t_{r}\right)\right] \\
\rightarrow & \frac{\left[\mu^{p}(f) p !\right]^{r}[\Gamma(\beta) \Gamma(2-\beta)]^{p r / 2}}{2^{p r / 2}(p r / 2) !} \sum \int_{0}^{t_{1}} d s_{1} \ldots \int_{0}^{t_{r}} d s_{r}\left|s_{u(1)}-s_{v(1)}\right|^{\beta-1} \ldots\left|s_{u(p r / 2)}-s_{v(p r / 2)}\right|^{\beta-1} .
\end{aligned}
$$

This is the joint $r$-th moment formula for a (non-standardized) Hermite process [25, Remark 4.4.2] ( $p$ there corresponds to $r$ here, and $k$ there correspond to $p$ here.)

Now we are left to take another expectation in (65). The conclusion will follow if the uniform integrability of

$$
\left(\mathbb{E}_{N}\left[S_{n}^{*}\left(t_{1}\right) \ldots S_{n}^{*}\left(t_{r}\right)\right], n \geq 1\right)
$$

holds. To show this, we consider the boundedness of

$$
\mathbb{E}\left[\left(\mathbb{E}_{N}\left[S_{n}^{*}\left(t_{1}\right) \ldots S_{n}^{*}\left(t_{r}\right)\right]\right)^{2}\right] \leq \mathbb{E}\left[\mathbb{E}_{N}\left[S_{n}^{*}\left(t_{1}\right)^{2} \ldots S_{n}^{*}\left(t_{r}\right)^{2}\right]\right] .
$$

In view of (66) and (67), we have

$$
\mathbb{E}_{N}\left[S_{n}^{*}\left(t_{1}\right)^{2} \ldots S_{n}^{*}\left(t_{r}\right)^{2}\right] \leq c w_{n}^{-2 q} \sum_{m=p}^{p r}\left(\begin{array}{c}
N_{n} \\
m
\end{array}\right) .
$$

Using the fact $\mathbb{E}_{N}\left[N_{n}\left(N_{n}-1\right)\left(N_{n}-m+1\right)\right]=Q^{m} w_{n}^{m}$, we see that $\mathbb{E}\left[\mathbb{E}_{N}\left[S_{n}^{*}\left(t_{1}\right) \ldots S_{n}^{*}\left(t_{r}\right)\right]^{2}\right]$ is bounded.

Proof of Theorem 3.3. Note that the normalization $\left(a_{n}\right)$ chosen in (22) ensures that the limit has variance $\mu^{p}(f)$ at $t=1$, which can be verified with Corollary 3.1 and 25, Proposition 2.2.5]. Note also that in view of the relation between $\left(b_{n}\right)$ and $\left(w_{n}\right)$ in (12), the normalization $\left(\frac{w_{n}^{p / 2} n}{b_{n}^{p}}\right)$ in Proposition 4.6 and the normalization $\left(a_{n}\right)$ in (22) are asymptotically equivalent up to a constant. So in the case $\rho=\rho_{0}$ as in Lemma 4.3 the convergence of finite-dimensional distributions follows from Proposition 4.6, the moment determinancy of a distribution living in the first and second Wiener chaos [32], and the fact that the multidimensional moment determinacy follows from the moment determinacy of the marginals (31, Theorem 14.6], see also the proof of Theorem 3.2 above). The extension from $\rho_{0}$ to general $\rho$ follows from Lemma 4.3 Tightness in $D[0,1]$ is a routine result in this long-range dependence regime (Corollary [3.1] 25, Proposition 2.2.5] and [9, Proposition 4.4.2]). 
Acknowledgment. The author would like to thank Takashi Owada and Yizao Wang for helpful discussions. The author would like to thank the anonymous referees for their careful reading and helpful suggestions which have lead to substantial improvements of the paper.

Shuyang Bai

Department of Statistics

University of Georgia

310 Herty Drive,

Athens, GA, 30602, USA.

bsy9142@uga.edu

\section{References}

[1] J. Aaronson. An introduction to infinite ergodic theory. Number 50. American Mathematical Soc., 1997.

[2] S. Bai. Representations of Hermite processes using local time of intersecting stationary stable regenerative sets. To appear in Journal of Applied Probability, 2019. arXiv preprint arXiv:1910.07120.

[3] S. Bai and M. S. Taqqu. Limit theorems for long-memory flows on Wiener chaos. Bernoulli, 26(2): 1473-1503, 2020.

[4] S. Bai, T. Owada, and Y. Wang. A functional non-central limit theorem for multiple-stable processes with long-range dependence. Stochastic Processes and their Applications, 130(9):5768-5801, 2020.

[5] N. Bingham, C. Goldie, and J. Teugels. Regular Variation. Encyclopedia of Mathematics and Its Applications. Cambridge University Press, 1989.

[6] Z. Chen and G. Samorodnitsky. Extremal clustering under moderate long range dependence and moderately heavy tails. arXiv preprint arXiv:2003.05038, 2020.

[7] Z. Chen and G. Samorodnitsky. Extreme value theory for long-range-dependent stable random fields. Journal of Theoretical Probability, 33(4):1894-1918, 2020.

[8] R. Dobrushin and P. Major. Non-central limit theorems for non-linear functional of Gaussian fields. Probability Theory and Related Fields, 50(1):27-52, 1979.

[9] L. Giraitis, H. Koul, and D. Surgailis. Large Sample Inference for Long Memory Processes. World Scientific Publishing Company Incorporated, 2012.

[10] S. Gouëzel. Correlation asymptotics from large deviations in dynamical systems with infinite measure. In Colloquium Mathematicum, volume 125, pages 193-212. Instytut Matematyczny Polskiej Akademii Nauk, 2011.

[11] A. B. Hajian and S. Kakutani. Weakly wandering sets and invariant measures. Transactions of the American Mathematical Society, 110(1):136-151, 1964.

[12] K. Itô. Multiple Wiener integral. Journal of the Mathematical Society of Japan, 3(1):157-169, 1951.

[13] S. Janson. Gaussian Hilbert Spaces, volume 129. Cambridge university press, 1997.

[14] P. Jung, T. Owada, and G. Samorodnitsky. Functional central limit theorem for a class of negatively dependent heavy-tailed stationary infinitely divisible processes generated by conservative flows. The Annals of Probability, 45(4):2087-2130, 2017.

[15] O. Kallenberg. Foundations of Modern Probability. Springer Science \& Business Media, second edition, 2002. 
[16] O. Kallenberg. Random Measures, Theory and Applications. Springer, 2017.

[17] M. Kesseböhmer and M. Slassi. Limit laws for distorted critical return time processes in infinite ergodic theory. Stochastics and Dynamics, 7(01):103-121, 2007.

[18] K. Krickeberg. Strong mixing properties of Markov chains with infinite invariant measure. In Proc. Fifth Berkeley Sympos. Math. Statist. and Probability (Berkeley, Calif., 1965/66), volume 2, pages 431-446, 1967.

[19] C. Lacaux and G. Samorodnitsky. Time-changed extremal process as a random sup measure. Bernoulli, 22(4):1979-2000, 2016.

[20] I. Melbourne and D. Terhesiu. Operator renewal theory and mixing rates for dynamical systems with infinite measure. Inventiones mathematicae, 189(1):61-110, 2012.

[21] T. Owada. Limit theory for the sample autocovariance for heavy-tailed stationary infinitely divisible processes generated by conservative flows. Journal of Theoretical Probability, 29(1):63-95, 2016.

[22] T. Owada and G. Samorodnitsky. Maxima of long memory stationary symmetric alpha-stable processes, and self-similar processes with stationary max-increments. Bernoulli, 21(3):1575-1599, 2015.

[23] T. Owada and G. Samorodnitsky. Functional central limit theorem for heavy tailed stationary infinitely divisible processes generated by conservative flows. The Annals of Probability, 43(1):240-285, 2015.

[24] G. Peccati and M. Taqqu. Wiener Chaos: Moments, Cumulants and Diagrams: a Survey With Computer Implementation. Springer Verlag, 2011.

[25] V. Pipiras and M. Taqqu. Long-Range Dependence and Self-Similarity, volume 45. Cambridge University Press, 2017.

[26] J. Rosinski. On series representations of infinitely divisible random vectors. The Annals of Probability, pages 405-430, 1990.

[27] J. Rosinski and G. Samorodnitsky. Product formula, tails and independence of multiple stable integrals. Advances in stochastic inequalities (Atlanta, GA, 1997), 234:169-194, 1999.

[28] G. Samorodnitsky. Stochastic Processes and Long Range Dependence, volume 26. Springer, 2016.

[29] G. Samorodnitsky and J. Szulga. An asymptotic evaluation of the tail of a multiple symmetric $\alpha$-stable integral. The Annals of Probability, pages 1503-1520, 1989.

[30] G. Samorodnitsky and Y. Wang. Extremal theory for long range dependent infinitely divisible processes. The Annals of Probability, 47(4):2529-2562, 2019.

[31] K. Schmüdgen. The moment problem, volume 9. Springer, 2017.

[32] E. Slud. The moment problem for polynomial forms in normal random variables. The Annals of Probability, pages 2200-2214, 1993.

[33] J. Szulga. Multiple stochastic integrals with respect to symmetric infinitely divisible random measures. The Annals of Probability, pages 1145-1156, 1991.

[34] M. Taqqu. Convergence of integrated processes of arbitrary Hermite rank. Probability Theory and Related Fields, 50(1):53-83, 1979. 